NASA

Technical

Paper

2839

November 1988

\title{
Interactive Orbital \\ Proximity Operations \\ Planning System
}

Arthur J. Grunwald

and Stephen R. Ellis

(AASA-TE-2839) IATEEACTIVE CEEIIAL

EFCXIEIIY CEELAILCAS EIAANIAG EYSTER (DASA)

$4 \mathrm{E}$
CSCI OSH
$N E 9-1 \in C 3 y$

Unclas C $19172 \%$ 
NASA

Technical

Paper

2839

1988

\section{Interactive Orbital Proximity Operations Planning System}

Arthur J. Grunwald and Stephen R. Ellis

Ames Research Center

Moffett Field, California 


\title{
INTERACTIVE ORBITAL PROXIMITY OPERATIONS PLANNING SYSTEM
}

\author{
Arthur J. Grunwald* and Stephen R. Ellis** \\ Ames Research Center
}

SUMMARY

An interactive graphical proximity operations planning system has been developed, which allows on-site design of efficient, complex, multiburn maneuvers in a dynamic multispacecraft environment. Maneuvering takes place in and out of the orbital plane. The difficulty in planning such missions results from the unusual and counterintuitive character of orbital dynamics and complex time-varying operational constraints. This difficulty is greatly overcome by visualizing the relative trajectories and the relevant constraints in an easily interpretable graphical format, which provides the operator with immediate feedback on design actions. The display shows a perspective bird's-eye view of a Space Station and co-orbiting spacecraft on the background of the Station's orbital plane. The operator has control over two modes of operation: (1) a viewing system mode, which enables the exploration of the spatial situation about the Space Station and thus the ability to choose and zoom in on areas of interest; and (2) a trajectory design mode, which allows the interactive "editing" of a series of way points and maneuvering burns to obtain a trajectory that complies with all operational constraints. A first version of this display has been completed. An experimental program is planned in which operators will carry out a series of design missions which vary in complexity and constraints.

\section{INTRODUCTION}

The future Space Station environment will include a variety of spacecraft co-orbiting in close vicinity. Usually, these spacecraft will be placed in a stable location with respect to the Space Station; i.e., they will share the same circular orbit. However, some missions will require repositioning of or transfers to and from these spacecraft. In this case complex maneuvers are anticipated which involve a variety of spacecraft not necessarily located at stable locations, and thus there

\footnotetext{
*National Research Council Senior Research Associate at NASA Ames Research Center 1985-1987 and Senior Lecturer at the Department of Aeronautical Engineering, Technion-Israel Institute of Technology, Haifa, Israel. Partially supported by a grant of the National Research Council.

**Research Scientist at NASA Ames Research Center and Assistant Professor at the Department of Physiological Optics at U.C. Berkeley, CA, USA.
} 
will be relative motion between them. (See appendix for an expanded treatment of the subject.

The multivehicle environment poses new requirements which do not exist in conventional mission scenarios. The conventional scenarios involve proximity operations between only two vehicles. In these two-spacecraft missions, the scenario is optimized through considerable advanced planning and executed at the time of the actual mission. However, since the set of possible scenarios in a multivehicle environment is virtually unlimited, the future Space Station environment will create scenarios that might not have been precomputed and that will have to be planned and executed on-site. This will require an on-site planning tool which allows, through a fast interactive process, the creation of a fuel-efficient maneuver which meets all constraints set by safety rules.

The difficulties encountered in planning and carrying out orbital maneuvers originate from several causes. The first is the counterintuitive character of orbital motions as experienced in a relative reference frame. The orbital motions are expressed in a coordinate frame attached to the Space Station and represent relative rather than absolute motions. It would be intuitively assumed that a thrust in the forward direction, i.e., in the direction of the orbital velocity vector, would result in a straightforward motion. However, after several minutes, orbital mechanics forces will dominate the motion pattern and move the spacecraft upward, i.e., to a higher orbit. This will result in a backward relative motion, since objects in a higher orbit move more slowly. Thus, a forward thrust has an effect opposite from that intended.

A second cause of the difficulty is the different and unusual way in which orbital maneuvering control forces are applied. In atmospheric flight, control forces are applied continuously to correct for randomly appearing atmospheric disturbances, or to compensate for atmospheric drag. In contrast, spaceflight in the absence of atmospheric disturbances has a near-deterministic character. Therefore, spaceflight is mainly unpowered along a section of an orbit with certain characteristics. By applying impulse-type maneuvering forces at a given way point, the characteristics of the orbit are altered. After application of the maneuvering force, the spacecraft will coast along on the revised orbit until the next way point is reached.

Third, multivehicle orbital missions are subject to stringent safety constraints, such as clearance from existing structures, allowable approach velocities, angles of departure and arrival, and maneuvering-burn restrictions due to plume impingement. Design of a fuel-efficient trajectory which satisfies these constraints is a nontrivial task.

It is clear that visualization of the relative trajectories and control forces in an easily interpretable graphical format will greatly improve the feel for orbital motions and control forces and will provide direct feedback of the operator's control actions. Furthermore, visualization of the constraints in a symbolic graphical format will enable an interactive graphical trajectory design in which, in each iteration step, the design is modified until all constraints are satisfied. 


\section{Definition of Coordinate Systems and Orbit ${ }^{1}$}

The trajectory of a spacecraft orbiting a given planet is determined by a set of six orbital parameters. In figures $1(a)$ and $1(b)$ the orbit is visualized. The orientation of the plane of the orbit in the inertial system $x^{i} y^{i} z^{i}$ is determined by the inclination $i$ and the longitude $\Omega$ of the ascending node AN (see fig. 1(a)). In the orbital plane the shape of the orbit can be either circular, elliptic, parabolic, or hyperbolic, depending on the total energy. In our case all orbits are either circular or elliptic. Figure 1(b) shows the shape of the orbit in the orbital plane. The ellipse is located with one of its foci $F_{1}$ at the Earth center of mass. The orientation of the ellipse is determined by the angle of the line of apsides $w$, which is the angle between the ellipse major axis and the line 0 - AN (which is the intersection of the orbital plane with the horizontal $x^{i} y^{i}$ plane). The ellipse is characterized by the semimajor axis a and the eccentricity e. For a circular orbit the eccentricity is zero and a will be the radius of the circle. For the Earth orbit the point closest to Earth is the perigee PE and the point farthest away is the apogee AP. Finally, the position of the spacecraft in orbit is fully determined by the time of perigee passage $t=T$, or equivalently, by the angle of the true anomaly $v$.

In our case all orbital maneuvering takes place about the Space Station. The station is stabilized in circular orbit with inclination $i=28.5^{\circ}$ with respect to the equatorial plane, an altitude of $h=480 \mathrm{~km}$ above the Earth surface, and an orbital velocity of about $V_{0}=7,623 \mathrm{~m} / \mathrm{sec}$. Trajectory design can be greatly simplified by expressing the positions and velocities of co-orbiting spacecraft relative to a Space Station-based coordinate system. This system $x^{\circ} y^{\circ}{ }^{\circ}$ has its origin at the center of mass of the station, is oriented with the $x^{0} y^{0}$ plane locally level with the surface of the Earth, with the $x^{\circ}$-axis in the direction of the station's orbital velocity vector, and with the $z^{\circ}$-axis pointing toward the center of the Earth. Thus, the $x^{\circ} z^{\circ}$ plane constitutes the orbital plane.

The orbital parameter set of a co-orbiting spacecraft can be fully determined by measuring the momentary relative position and velocity with respect to the Space Station system. These measurements can be performed with great accuracy with Space Station-based sensors. The equations for computing the orbital parameters of a co-orbiting spacecraft from its measured position and motion relative to the Space Station are derived from references $1-3$ and are given in the appendix.

The coordinate system used here, as well as that in the source code which it describes, is the same used in references 1 and 2. It differs from that used in many NASA documents in that $+R-b a r$ is away from the Earth rather than toward it. 


\section{Definition of V-Bar, R-Bar, Stationary and Nonstationary Locations}

The $V$-bar is defined as a section of the circular orbit, followed by the center of mass of the Space Station, and the R-bar is defined as the radial line moving outward from the Earth center through either the spacecraft or the Space Station (see fig. 2). The most appropriate way to determine the in-orbital-plane position of a spacecraft relative to the Space Station would be in terms of $s$ and $r$, where $s$ is the distance measured along the circular $V$-bar between the Space Station and the spacecraft's R-bar, and $r$, the distance of the spacecraft below the V-bar, measured along the spacecraft's R-bar (see fig. 2). The velocity vector of the spacecraft relative to the Space Station is denoted by $\underline{v}$, and its components in the $\mathrm{V}$-bar and R-bar direction are denoted by $\dot{s}$ and $\dot{r}$, respectively. Equivalently, the vector $\underline{v}$ can be expressed as the magnitude $v$ and its angle with the $V$-bar $\alpha$ (see fig. 2). Since the range of operation about the Space Station is less than several kilometers, the $V$-bar can be considered to be straight and equivalent to the $x^{\circ}$ axis. Thus $x^{\circ} \approx s$ and $z^{\circ} \approx-r$. The out-of-plane displacement is $y^{0} \equiv w$. The relative position is denoted concisely by the vector $\underline{x}$.

A spacecraft, located on the $\mathrm{V}$-bar and with zero velocity relative to the Space Station, will, on the assumption of identical drag characteristics, be stationary with respect to the Space Station, since it has the same orbital radius $R_{0}$ and period as the Space Station. A spacecraft with radial separation, i.e., above or below the V-bar, will not be stationary relative to the Space Station. A spacecraft in circular orbit above the $V$-bar, with radius $R=R_{0}+r$, will tend to fall behind, i.e., move backward with respect to the orbital flight direction. In contrast, a spacecraft in circular orbit below the V-bar will tend to pull ahead. This relative motion is caused by a difference in mean angular orbital rate, or mean motion, between the spacecraft and the space Station. It is well known from Kepler's third law that the higher the orbit, the slower the mean motion. If the mean motion of the spacecraft in $\mathrm{rad} / \mathrm{sec}$ is denoted by $\mathrm{n}$, and of the space station by $n_{0}$, the difference in mean motion $\Delta n$ is approximated by

$$
\Delta \mathrm{n} \equiv \mathrm{n}-\mathrm{n}_{0} \approx-\frac{3}{2}\left(\frac{\mathrm{r}}{\mathrm{R}_{0}}\right) \mathrm{n}_{0}
$$

It is seen from equation (1) that after one Space Station orbit, the spacecraft has fallen behind or pulled ahead by the distance $3 \pi r$ meters, where $r$ is the distance above or below the $\mathrm{V}$-bar in meters.

Even when the relative velocity of a spacecraft with radial separation is initially zero, it will not be stationary relative to the Space Station. The shapes of the orbits for a location above and below the V-bar with an initial radial separation $r$ and an initial zero relative velocity are shown in figure $3(\mathrm{a})$, and the corresponding relative trajectories are shown in figure $3(b)$. The spacecraft above the $V$-bar is initially at perigee and the spacecraft below the V-bar is at apogee. The spacecraft above the $V$-bar moves initially radially outward away from the Earth, and after that starts to fall behind. In contrast, the spacecraft below the V-bar 
will initially move radially inward toward Earth and after that pull ahead. The eccentricity of the orbit and the difference in mean motion between spacecraft and Space Station are given by

$$
\begin{aligned}
& e=\left|1-\frac{\mathrm{R}}{\mathrm{r}_{0}}{ }^{3}\right| \approx\left|3 \frac{\mathrm{r}}{\mathrm{R}_{0}}\right| \\
& \Delta \mathrm{n} \approx-6\left(\frac{\mathrm{r}}{\mathrm{R}_{0}}\right) \mathrm{n}_{0}
\end{aligned}
$$

and the relative motion starting from $t=t_{0}$ is approximated by

$$
\begin{aligned}
& s(t)=s\left(t_{0}\right)-6 r\left[n_{0}\left(t-t_{0}\right)+\sin n\left(t-t_{0}\right)\right] \\
& r(t)=r\left(t_{0}\right)+3 r\left[1-\cos n\left(t-t_{0}\right)\right]
\end{aligned}
$$

Equations (4a) and (4b) are obtained by solving the general equations for relative motion given in the appendix for the particular case in which $r\left(t_{0}\right) \neq 0$ and $\dot{s}\left(t_{0}\right)$ and $\dot{r}\left(t_{0}\right)$ are zero. It is clear from equations (4a) and (4b) that after one Space Station orbit the spacecraft is again back on its original radius $R$, but it has fallen behind or pulled ahead by the distance $12 \pi r$ meters where $r$ is the distance above or below the $\mathrm{V}$-bar in meters. The maximum deviation in $r$-direction from $r\left(t_{0}\right)$ occurs at one-half orbit and is $6 r$ meters.

\section{Typical Maneuvers--V-Bar and R-Bar Burn}

Consider a spacecraft located at the $V$-bar, and thus at a stationary position relative to the Space Station. A small maneuvering burn in the direction of the positive R-bar will cause a small component $v$ which will result in a small change in the direction of the orbital velocity vector. This will alter the parameters of the orbit. The orbit will become elliptical and the spacecraft will be at apogee after one quarter of an orbital period. In figures $4(a)$ and $4(b)$ the shape of the orbit and the corresponding relative motion trajectory is shown. The relative trajectory has a closed elliptical shape and after one orbit the spacecraft will return to its original location. The reason for this is that the radial burn did not significantly alter the magnitude of $v_{0}$ and thus the total energy and mean motion did not change. The eccentricity, difference in mean motions and relative motion for an R-bar burn are given by

$$
\begin{aligned}
e & =\left|\frac{v}{V_{0}}\right| \\
\Delta n & \approx 0
\end{aligned}
$$




$$
\begin{aligned}
& s(t) \approx s\left(t_{0}\right)-2 R_{0}\left(\frac{v}{V_{0}}\right)\left[1-\cos n_{0}\left(t-t_{0}\right)\right] \\
& r(t) \approx R_{0}\left(\frac{v}{V_{0}}\right) \sin n_{0}\left(t-t_{0}\right)
\end{aligned}
$$

Equations (7a) and ( $7 b)$ are obtained by solving the general equations in the appendix for the case $r\left(t_{0}\right)=0, \dot{s}\left(t_{0}\right)=0$, and $\dot{r}\left(t_{0}\right) \equiv v \neq 0$. Since $n_{0}=v_{0} / R_{0}$, it is seen from equations (7a) and (7b) that for a positive $R-b a r$ burn of $1 \mathrm{~m} / \mathrm{sec}$, after half the orbital period of the Space Station, the spacecraft is again at the $V$-bar, but has fallen behind the distance $4 / n_{0} \approx 3,598 \mathrm{~m}$. The maximum deviation from the $V$-bar occurs at one-fourth and three-fourths of an orbital period of the Space Station and is $1 / \mathrm{n}_{0} \approx 899 \mathrm{~m}$.

In contrast to the R-bar burn, a maneuvering burn along the V-bar will alter the magnitude of $v_{0}$ by the amount $v$ and will therefore alter the total energy. Figures $5(\mathrm{a})$ and $5(\mathrm{~b})$ show the shape of the orbit and the corresponding relative motion trajectory. For a burn in the positive V-bar direction, the spacecraft will initially move forward, but later on it will gain altitude and fall behind. The opposite is the case for a burn in negative $V$-bar direction. Here the spacecraft will initially move backward, but later on drop altitude and pull ahead. For a positive burn the spacecraft is initially at perigee and for a negative burn at apogee. The eccentricity, difference in mean motions, and relative motion for a V-bar burn are given by

$$
\begin{aligned}
e & =2\left|\frac{v}{V_{0}}\right|+\left(\frac{v}{v_{0}}\right)^{2} \approx 2\left|\frac{v}{V_{0}}\right| \\
\Delta n & \approx-3 \frac{v}{R_{0}} \\
s(t) & \approx s\left(t_{0}\right)+R_{0}\left(\frac{v}{V_{0}}\right)\left[-3 n_{0}\left(t-t_{0}\right)+4 \sin n\left(t-t_{0}\right)\right] \\
r(t) & \approx 2 R_{0}\left(\frac{v}{v_{0}}\right)\left[1-\cos \left(t-t_{0}\right)\right]
\end{aligned}
$$

Equations (10a) and (10b) are obtained by solving the general equations in the appendix for the case $r\left(t_{0}\right)=0, \dot{s}\left(t_{0}\right) \equiv v \neq 0$, and $\dot{r}\left(t_{0}\right)=0$. Equations (10a) and $(10 \mathrm{~b})$ show that for a positive $V$-bar burn of $1 \mathrm{~m} / \mathrm{sec}$, after one orbital period of the Space Station, the spacecraft has fallen behind the distance $6 \pi / \mathrm{n}_{0} \approx 16,957 \mathrm{~m}$ and the maximum distance from the $\mathrm{V}$-bar is $4 / \mathrm{n}_{0} \approx 3,598 \mathrm{~m}$. 


\section{Limitations of Conventional Techniques}

It is clear from the above examples that orbital motion can be complex and highly counterintuitive. A burn toward the target might have the opposite result than intended. Motion is particularly complex and difficult to visualize for a combined burn at a nonstationary location. It is therefore very useful to graphically visualize the relative motion trajectories. This is true in particular for on-site interactive trajectory design in which immediate graphical feedback on the results of maneuvering burns is essential.

The presently used conventional techniques are well established and rely in most cases on visual contact and the use of a V-bar or R-bar reference in a Crewman Optical Alignment Sight (COAS) (ref. 4). In a V-bar approach toward a target in positive $V$-bar direction, the initial burn is made in a direction slightly depressed downward with respect to the $V$-bar. After a short while, the spacecraft will ascend and cross the V-bar. At the V-bar crossing, a small downward R-burn is initiated which again depresses the spacecraft below the $V$-bar. This process is repeated several times. The spacecraft thus proceeds along the V-bar in small hops until the target is reached. A similar approach is possible along the R-bar. However, these scenarios are highly restricted in their complexity and are not likely to satisfy the complex constraints of the multivehicle environment.

\section{DESCRIPTION OF THE TECHNIQUE}

\section{Purpose of Orbital Planning System}

The purpose of the interactive orbital planning system is to enable the operator to design an efficient, complex, multiburn maneuver, subject to the stringent safety constraints of the future dense traffic environment of the Space station, which enables a chaser to rendevous with a target spacecraft in a given timespan. The constraints include clearances from structures, relative velocities between spacecraft, angles of departure and arrival, approach velocity, and plume impingement. Because of the complexity and counterintuitiveness of orbital motion, and the demands to satisfy strict safety rules and constraints, fuel-efficient trajectory design will be a complex and difficult task. The basic idea underlying the system is to present the maneuver as well as the relevant constraints in an easily interpretable graphical format. This format provides the operator with immediate feedback on the results of design actions, and allows for close interaction with the system. In an iterative process, the operator will keep changing the design until all constraints are met. The methods for enabling interactive trajectory design and visualization of constraints are discussed in detail hereafter. 


\section{Illustration of a Three-Burn Maneuver}

An illustrative example of a three-burn maneuver is shown schematically in figure 6. The trajectory originates from relative position $A$ at $t$ ime $t=t_{0}$, and is composed of two way points $B$ and $C$ which specify the location in Space Station coordinates through which the chaser spacecraft will pass at a given time. At a way point the orbital maneuvering system or other reaction control system is activated, creating a thrust vector of given magnitude for a given duration, in a given direction in orbital plane or out of the orbital plane. The duration of the burn is considered very short in comparison with the total duration of the mission. In the orbital dynamics computations this means that a maneuvering burn can be considered as a velocity impulse which alters the direction and magnitude of the instantaneous orbital velocity vector of the spacecraft.

Since the initial location $A$ is not necessarily a stationary point, the magnitude and direction of the relative velocity of the chaser at point $A$ is determined by the parameters of its orbit. If no maneuvering burn is to be initiated at $t=t_{0}$, the chaser will continue to follow the relative trajectory 1 , subject to the parameters of its original orbit (see dotted line in fig. 6). However, a maneuvering burn at $t=t_{0}$ will alter the original orbit; thus the chaser will follow the relative trajectory 2 , subject to the parameters of the new orbit.

In figure $6, \underline{v}_{1}$ and $\underline{v}_{2}$ indicate the relative velocity vector of the chaser just before and after the maneuvering burn, where $\underline{v}_{1}$ and $\underline{v}_{2}$ are tangential to the relative trajectories 1 and 2 , respectively. The vector difference between $\underline{v}_{1}$ and $\underline{v}_{2}, \underline{v}_{a}$ is the velocity change initiated by the burn, and corresponds to the direction and magnitude or duration at which the orbital maneuvering system is activated. Likewise, at way point $B$ the burn $\underline{v}_{b}$ alters the orbit to orbit 3 .

Location $\mathrm{C}$ is the terminal way point and in this case is the location where the target will arrive at $t=t_{f}$. Since the target has an orbit of its own, orbit 4 , it will have a terminal velocity at $t=t_{f}$. The relative velocity between target and chaser is the vector difference between $\underline{v}_{3}$ and $\underline{v}_{4}, \underline{v}_{c}$. This vector determines the retro-burn that is needed at the target location to bring the relative velocity between chaser and target to the minimum required for the docking operation.

\section{Inverse Method of Solving Orbital Motion}

Interactive trajectory design demands that the operator is given free control over the positioning of way points. However, the input variables of the equations of orbital motion given in the Appendix are the magnitude and direction of the burn, rather than the position of way points. Therefore an inverse method is required to compute the values of a burn necessary to arrive at a given way point positioned by the operator. This method is outlined here.

The equations in the appendix show how the orbital parameters of a co-orbiting spacecraft can be computed from its momentary position and velocities, relative to 
the Space Station. Thus, for a given initial relative position $A$ with $\underline{x}\left(t_{0}\right)$ and an initial relative velocity $\underline{v}\left(t_{0}\right)$ at time $t=t_{0}$, the relative position and velocities of a way point at time $t=t_{1}$ can be computed. However, a maneuvering burn at $t=t_{0}$ will cause a change in the direction and magnitude of the relative velocity vector $\underline{v}\left(t_{0}\right)$. As a result, the position of the way point at time $t=t_{1}$, $\underline{x}\left(t_{1}\right)$ will change as well.

Consider $v_{a}$ and $a_{a}$ to be the magnitude and direction of the velocity change due to the maneuvering burn. Then the relative position and velocity at $t=t_{1}$, $\underline{x}\left(t_{1}\right)$, will be a complex nonlinear function of $v_{a}$ and $a_{a}$. Consider now that the operator is given direct control over $v_{a}$ and $\alpha_{a}$ by slaving these variables directly to the $x$ and $y$ motions of the mouse, respectively. A displacement of the mouse in either $x$ or $y$ direction will result in a complex nonlinear motion pattern of $\underline{x}\left(t_{1}\right)$. Furthermore, this motion pattern will change with the initial conditions. This arrangement is highly undesirable in an interactive trajectory design process in which the operator must have direct and unconstrained control over the positioning of way points.

It is therefore essential to give the operator direct control over the position of way points rather than over the magnitude and direction of the burn. The inverse method by which this is accomplished computes the magnitude and direction of the burn required to bring the spacecraft from initial location $\underline{x}\left(t_{0}\right)$ at $t=t_{0}$ to the way point $\underline{x}\left(t_{1}\right)$ at $t=t_{1}$. A Newton-Raphson method has been employed to solve this inverse problem. The operator commands the position of a way point by means of the $x-y$ motions of the mouse. The algorithm starts with an initial guess of $v_{a}$ and $\alpha_{a}$. These values yield a computed way point which is usually different from the commanded one. At each program update the values of $v_{a}$ and $a_{a}$ are adjusted to bring the computed way point closer to the commanded one. On the average about three or four iterations are required to bring the difference between the computed and commanded way point effectively to zero. As the operator moves the commanded way point around in the orbital plane, the algorithm tracks the commanded way point by continuously making appropriate adjustments in $v_{a}$ and $a_{a}$. Because of this continuous adjustment, the deviation between commanded and computed way point will remain relatively small and the Newton-Raphson scheme will operate in the vicinity of the optimum. The advantage of the Newton-Raphson scheme is that convergence with this second-order technique is the best in the near vicinity of the optimum. Since the program update rate is about $15 \mathrm{~Hz}$, convergence is very fast and the computed way point is virtually indistinguishable from the commanded one.

\section{Concept of the Active Way Point}

Although a trajectory may be composed of several way points, only one way point at a time, the active way point, is controlled by the operator. While the position and time-of-arrival of the active way point can be varied, the position and time-ofarrival of all other way points remain unchanged. However, variations in the active way point will cause changes in the trajectory sections and way-point maneuvering burns just preceding and just following the active way point. The on-line solution 
of the inverse algorithm enables these changes to be visualized almost instantaneously and provides the operator with on-line feedback on design actions.

Although impingement constraints and approach velocity limits exist for all way points, it is useful to limit the computation and display of these constraints to the active way point only. This arrangement simplifies and speeds up system update computations and minimizes the symbology shown on the display. The justification for this is that the operator's attention is mainly allocated to the active way point and its near vicinity. In a subsequent design iteration the operator may shift the activation to a different way point and again verify whether all constraints are met.

Since impingement constraints and approach velocity limits mainly relate to the target craft, it is useful to visualize the position of the target on the target trajectory, corresponding to the time of arrival at the active way point. Both the active way point, and the target position at active way-point time should be clearly distinguishable from other way points by conspicuous markings, highlighting, or blinking.

\section{Process of Way-Point Editing}

The trajectory-design process involves changes in existing way points, addition of new points, or deletion of existing undesired points. An illustrative example of this way-point editing process is shown in figure 7 . In the program the way points are managed by a way-point stack, which includes an up-to-date sequential list of the position $\underline{x}$, the time-of-arrival $t$, and the relative velocity $\underline{v}$ just after initiating the burn, of all way points.

Figure 7(a) shows two way points, the initial point $\underline{x}_{0}$ and the terminal point $\underline{x}_{1}$. The initial way point is defined by the initial conditions of the situation and cannot be activated or changed by the operator. The terminal way point $\underline{x}_{1}$ is thus the active way point which can be changed. The corresponding way-point stack is shown on the right. The active way-point box is drawn in bold. The relative velocity stack shows only the velocity $\underline{v}_{0}$, which is the relative required velocity just after the burn at way point 0 , computed by the inverse algorithm, and required to reach point $\underline{x}_{1}$ at time $t_{1}$.

Figure 7(b) shows the addition of a new way point. The new way point is added half-way on the trajectory section just preceding the active way point. Thus its time-of-arrival is chosen to be $t=0.5\left(t_{i}+t_{i-1}\right)$, where $i$ is in this case 1 and relates to the stack before modification. The new position $\underline{x}_{1}$ and relative velocity $\underline{v}_{1}$ are computed by the forward method given in the appendix, by computing the orbital position at the new time $t$ using the existing orbital parameters previously computed with $\underline{x}_{0}, \underline{v}_{0}$, and $t_{0}$. The newly computed way-point position, time, and relative velocity are inserted between points 0 and 1 of the stack before modification, and the new way point is chosen to be the active one. The dotted lines in figure 7 indicate variables which are transferred without modification and the encircled variables are the newly computed ones. It is important to note that since 
the relative velocities $\underline{v}_{0}$ and $\underline{v}_{1}$ are matched to the required way points $\underline{x}_{1}$ and $\underline{x}_{2}$, respectively, the inverse algorithm does not need to make any adjustments.

Figure $7(c)$ shows the results of changes in the newly created way point on the way-point stack. Since $\underline{x}_{1}$ and $t_{1}$ are varied, the relative velocity at way-point 0 , $\underline{v}_{0}$, will be readjusted by the inverse algorithm and likewise the relative velocity $\underline{\mathrm{v}}_{1}$.

Figure $7(d)$ shows the creation of an additional new way point. Since the active way point prior to the addition was point 1, the new point is added half-way between points 0 and 1 and its position and relative velocity are computed with the forward method. The new values are inserted between points 0 and 1 of the stack before modification, and the new way point is again set to be the active one.

In figure $7(e)$ way point 2 is activated. Apart from the shift in active way point, the stack remains unchanged. The dotted line shows the direct-path section between point 1 and point 3 without the intermediate burn at point 2 . Deletion of way point 2 will remove this point from the stack and, after that, close the gap (see fig. $7(f)$ ). However, $\underline{v}_{1}$ has to be readjusted to fit the new direct-path section. Starting from the old incorrect value of $\underline{v}_{1}$, one can make the adjustment iteratively and on-line by the inverse algorithm.

\section{Constraints During Operations}

The multispacecraft environment will require strict safety rules regarding clearance from existing structures. Thus, spatial envelopes can be defined through which the spacecraft is not allowed to pass. These spatial constraints can be visualized on the display. The operator must be able to make a clear judgment as to whether the planned trajectory clears the spatial constraint. In case the trajectory does not clear the constraint, the operator must be able to decide whether to avoid the constraint through an in-plane maneuver or an out-of-plane maneuver. However, the operator is not always able to make these judgments on the basis of one-perspective aerial view or one-perspective projection. In this research, a graphical enhancement is used in which the spatial constraint is unambiguously presented in a time-axis display format. This format and its advantages are discussed in the "Description of the Display" section of this paper.

Restrictions on angles of departure and arrival may originate from structural constraints at the departure gate or from the orientation of the docking gate or grapple device at the target craft. Limits for the allowable angles of departure or arrival can be visualized on the display. In addition, the terminal approach velocity at the target might be limited by the characteristics of the grapple mechanism or the docking procedure. Limits for the allowable terminal approach velocity can be visualized as well.

Way point maneuvering burns are subject to plume impingement constraints. Hot exhaust gases of the orbital maneuvering systems may damage the reflecting surfaces of sensitive optical equipment such as telescopes or infrared sensors. Maneuvering 
burns toward these pieces of equipment are restricted in direction and magnitude; limits for the allowable direction and magnitude are a function of the distance to the equipment and plume characteristics. These limits can be visualized on the display.

Flight safety requires that the relative velocity between spacecraft is subject to approach velocity limits. In conventional docking procedures these limits were proportional to the range (refs. 5 and 6 ). A commonly used rule of thumb is to limit the relative approach velocity to 0.1 percent of the range. This conventional rule is quite conservative and originates from visual procedures in which large safety margins are taken into account to correct for human or system errors. Although the future traffic environment will be more complex, and will therefore demand larger safety margins, more advanced and reliable measurement and control systems will somewhat relax these demands. The effect of these developments on the allowable approach velocity limits is at present difficult to predict, and so is the margin for human error to be taken into account.

In this study, the relative approach velocity is defined as the component of the relative approach velocity vector between the two spacecraft along their mutual line of sight. The limit on this relative approach velocity is a function of the range between the spacecraft. This function will depend on the environment, the task, and the reliability of measurement and control equipment, and cannot be determined at this stage. In this study a simple proportional relation has been chosen. The approach velocity limit is visualized on the display as a circle indicating the minimum range between the two spacecraft allowed for the present approach velocity. If the target craft appears within this circle, the approach velocity limit has been violated.

\section{DESCRIPTION OF GRAPHICS SYSTEM AND LAYOUT OF THE DISPLAY AREA}

The system has been implemented on a Silicon Graphics IRIS 2400 Turbo Graphics Workstation with 24 bitplanes of display memory and with a 19-in. full-color display monitor with a display resolution of 1024 by 768 pixels. The program is named NAVIE (the Hebrew word for prophet) after the prophet Elijah, who was characterized as providing trustworthy future information. Operator interaction with the system is through a two-axis, three-button mouse.

The layout of the display area is shown in figure 8. The display area has been divided into four viewports, main area 1 (750 by 750 pixels) and areas 2, 3, and 4 (230 by 230 pixels each). Viewports 1, 3, and 4 provide information about the spatial situation around the Space Station, trajectories, constraints, and orbital maneuvering fuel use; and viewport 2 includes an eight-button function control panel. 


\section{DESCRIPTION OF DISPLAY PROGRAM CONTROL MODES}

The program operates in two basic modes. The first one, the system control mode, is engaged when no buttons are pressed. In this mode, motions of the mouse are controlling actions in windows 1 and 3 (such as viewpoint motion or way-point position). The second mode is devoted to function control and is activated by pressing the middle button of the mouse. When this button is held down, a cursor appears which can be positioned on one of the eight function buttons in area 2 . Selected buttons are highlighted and the function described by the button is activated only after the middle button is released.

While being switched from system control mode to function control mode, the system control actions are frozen and reactivated after return from the function control mode. Note that although the mouse values may have changed in the function control interval, the system control status remains unaltered. Likewise, the latest cursor position in the function control mode remains unchanged in the system control interval, although the mouse may have been moved. This feature is very useful when the same button is activated more than once. For example, this is done when toggling through a sequence. The toggling in this case can be done simply by pressing and releasing the middle mouse button several times, without the need for repositioning the cursor.

The system control mode has two submodes. The first one, the viewing system mode, relates to the main display which shows a perspective view of the Space Station and its surroundings on the background of the Station's orbital plane. In this viewing system mode, the operator is able to explore the spatial situation about the Space Station and thus choose a viewpoint location and viewing direction which focus and zoom in on the momentary area of interest. The second submode is the trajectory design mode, in which way points are selected, moved, added, and deleted in order to obtain a multiburn trajectory which complies with the given set of constraints.

\section{Viewing System Mode}

The geometry of the viewing situation is shown in figure 9 . The Space Stationbased coordinate system is $x^{0} y^{\circ} z^{\circ}$ with the $x^{\circ}$ axis coinciding with the orbital velocity vector, and the orbital plane is $x^{0} z^{0}$. Figure 9 shows the orientation of the viewing system $x^{e} y^{e} z^{e}$ relative to the space Station system. The viewing system has its origin at point $A$, the $x^{e}$ axis coincides with the viewing direction, and the image plane is perpendicular to the $x^{e}$ axis with the screen axes $y^{S}$ and $z^{s}$ parallel to $y^{e}$ and $z^{e}$. Point $B$ indicates the intersection of the viewing axis with the orbital plane. Although the viewing system position, point $A$, and angular orientation are defined by three displacements and three angles (which can all be controlled independently), it is useful to constrain the motion to the following three types. 
Tethered Motion- In the first type of motion the viewing system tethers about point $B$, which is kept fixed on the orbital grid, while the distance $d$ between points $A$ and $B$, which is the viewing range to point $B$, is kept constant. The tethered motion is controlled by the angles $\psi$ and $\theta$. The viewing axis $x$ and the axis $y^{e}$ are located at all times in the plane $P$ which passes through the point $B$ and rotates about the line $C^{\prime}$, which is parallel to the $x^{\circ}$ axis (the $\mathrm{V}$-bar). The line $\mathrm{BE}$ is also located in the plane $\mathrm{P}$, and is perpendicular to the Iine $\mathrm{CC}^{\prime} . \psi$ is the angle between the $\mathrm{y}^{\circ}$ axis and the line $\mathrm{BE}$, and $\theta$ is the angle between $B E$ and the $x^{e}$ axis. Thus, the angles $\psi$ and $\theta$ control the obliquity of viewing along the orbital plane in $z^{0}$ and $x^{\circ}$ directions, respectively. This tethered type of motion is very useful for the following reasons: (1) While the area of interest remains in the center of the display, it allows one to explore other possible areas of interest by bringing them into the viewport by changing the angles $\psi$ and $\theta$. (2) The line $\mathrm{CC}^{\prime}$ will appear on the screen at all times as a horizontal line through the center of the display, and will represent a line parallel to the $V$-bar. Thus, while the viewing direction may change, the direction of the $V$-bar is at all times recognizable as the horizontal line passing through the center of the display.

Translational Motion- The second type of motion relates to the position of point $\mathrm{B}$ in the orbital plane. Here the $\mathrm{x}^{\circ} \mathrm{z}^{\circ}$ coordinates of point $\mathrm{B}$ are varied, while $\psi, \theta$, and $d$ are kept constant. This type of translational motion enables the operator to move areas of interest to the center of the display.

Ranging Motion- In the third type of motion, all parameters are kept constant except for the range $d$. This ranging type of motion is useful after areas of interest are located and brought into the center of the display. Ranging-in on the area of interest allows this area to be studied in more detail.

In the viewing system mode, the operator can select either tethered motion, translational motion, or ranging motion by pressing the middle button. This onebutton control is useful since viewing system operations are naturally performed in a sequence of three steps. In the first step, areas of interest are selected. In the second step the selected area of interest is moved to the center of the display, and in the third step the area of interest is ranged-in on to obtain the required level of detail. Note that a ranging operation rather than a zoom operation has been chosen. This has been done to preserve the operator's awareness of the spatial location of the viewpoint. The ranging operation is consistent with the translational type of motion of point $B$, both of which are experienced by the operator as flying in three-dimensional space around the Space Station. In a zoom operation the viewpoint position remains unchanged and instead, the field of view is changed. However, since zooming is experienced as moving toward or away from the viewed object, this operation is bound to interfere with the operator's spatial orientation.

In addition to the control over the three types of motion, the viewing system mode has one additional control, a viewing system reset. This reset will return the viewing situation to a default setting which is defined in a program parameter file 
prior to the running of the program. The default viewing situation can be chosen to view the total area of interest from a more remote location.

\section{Trajectory Design Mode}

In the trajectory design mode, the operator has control over the seleotion, positioning, time of arrival, and addition and deletion of the way points which determine the trajectory. Two submodes exist in the trajectory design mode, the in-plane design mode, and the out-of-plane design mode. In the in-plane mode the mouse controls the $\mathrm{x}^{\circ} \mathrm{z}^{\circ}$ position of way points, while the out-of-plane position $y^{\circ}$ remains unchanged. In the out-of-plane mode the in-plane position remains unchanged and the out-of-plane position can be varied.

The design process starts with an initial configuration of way points. Usually there initially are two way points as in the example of the Way-Point Editing section. The terminal point $\underline{x}_{1}$ is the active way point. Time of arrival at the active way point is set to an initial value within the allowable timespan of the mission. The operator can increase or decrease the time of arrival at any active way point in preset steps by repeatedly pressing the right or left button, respectively, of the mouse. In this example a time-step of $10 \mathrm{sec}$ was found to be adequate. The time of arrival at the terminal way point is limited to the timespan of the mission, and the arrival time at intermediate way points is restricted by the timespan set by the neighboring points.

As outlined in the Way-Point Editing section, a convention is chosen in which a new way point is added to the trajectory section between the way point preceding the active way point, and the active way point itself, half-way on the time scale between the two way points. The newly added way point becomes the active one and can be moved to any desired location, and its time of arrival can be set to any value within the timespan determined by the neighboring way points. However, in some cases, it is useful to slide the new way point along the trajectory section directly connecting its neighboring way points. The position on this trajectory section is then determined by its time of arrival only. In this mode, the "lockedon-trajectory" mode, the time of arrival is slaved to the y-motions of the mouse.

The locked-on-trajectory mode is particularly useful for checking whether operational constraints between the spacecraft and the target or other nonstationary spacecraft are being violated. As the operator slides the way point along the trajectory, the corresponding target position slides along the target trace as well; conflicting situations, such as a too close fly-by, will be recognized immediately.

\section{Geometrical Enhancements--The Time-Axis Format}

The purpose of these enhancements is to resolve ambiguities in the spatial situation by processing the spatial information and presenting it in a different format. One such format is the time-axis display, which provides unambiguous 
qualitative and quantitative information about the out-of-plane situation and the spatial constraints.

The basic idea of the time-axis format is demonstrated in figures $10(a)-(c)$. From the perspective view of figure 10(a) alone it cannot be clearly determined whether the spatial constraint is violated or how the trajectory should be planned to avoid it. The view along the $z^{0}$ axis in figure $10(b)$ is even less clear, because of the curved character of the trajectory. In the time-axis format of figure $10(\mathrm{c})$ the out-of-plane deviation is plotted as a function of the traveled time along the path. The spatial constraints are visualized as follows. At each point on the traveled-time axis, at the corresponding location on the trajectory, a line is placed perpendicular to the orbital plane. Sections of this line which are within these constraints are identified and plotted on the time-axis display of figure 10 (c) as a set of vertical bars. Where the trajectory curve passes through these bars, the spatial constraints have been violated. From this display it can also be clearly determined whether the constraint should be avoided through an in-plane or an out-of-plane maneuver. Depending on the shape of the spatial constraint, an in-plane maneuver might vary the size and location of the constraint bars. For the rectangular constraint in figure 10(a) the bars might disappear entirely. In other cases, a small out-of-plane maneuver might be sufficient to avoid the spatial constraint bars.

The format of the time-axis display used in the program is shown in figure 11. The time axis is marked in quarters of an orbit. The shaded area represents the nighttime section of the orbit. This is useful in verifying requirements regarding the section of the orbit, i.e., day or night, in which a certain part of the mission has to take place. Both the target and the chaser trajectories are shown. It should be noted, however, that although the chaser and target share the same time axis, they relate to different spatial trajectories. Therefore the spatial-constraint bars are meaningful for the chaser trajectory only.

The time-axis display can be brought into the main viewport by a swap between viewports 1 and 3. This viewport swap feature is useful when certain parts of the time-axis display have to be viewed in more detail and the resolution of viewport 3 is insufficient.

\section{Symbolic Enhancements}

Visualization of Departure Constraints- Procedures at the departure gate might constrain the relative angle of departure and the magnitude of the departure burn. The in-plane constraints at the departure gate are visualized in figure 12. The size of the burn vector is made proportional to the burn magnitude. The departure constraints are satisfied if the burn vector is within the solid bracketed arc. This arc is specified by the arc center angle $\gamma_{0}$, the arc aperture $r$, and the arc radius $\varepsilon$. The scale-factor for visualizing the burn velocities in this example is $500 \mathrm{~m}$ length per $1 \mathrm{~m} / \mathrm{sec}$ burn. Note that maneuvering burns are expressed in terms 
of a velocity change rather than of a thrust force. The actual duration and thrust force of the burn depends on the spacecraft mass and the thruster characteristics.

In order to keep the display free from unnecessary symbology it is useful to present the constraint only when it is close to being violated. If the burn vector is within the area enclosed by the dotted line in figure 12, the constraint is not drawn. The radius of the dotted arc is $80 \%$ of $\varepsilon$ and the aperture angle is $10^{\circ}$ smaller than $\gamma$.

It should be noted that the situation in figure 12 relates to a stationary departure gate. The spacecraft trajectory in this case is aligned with the burn vector. For a departure gate which moves with respect to the Space Station system, this will not be the case. In this case the burn vector will signify the relative direction of departure with respect to the moving gate, rather than with respect to the Space station. But this is just the vector which is subject to the departure constraints and not the velocity vector of the trajectory, which is relative to the Space station. Therefore, the symbology is valid for departure from a stationary as well as nonstationary gate.

The out-of-plane constraint at the departure gate is visualized in figure 11. The initial out-of-plane component of the burn vector has to be within the impingement constraint brackets. If the burn magnitude is less than $80 \%$ of the allowed maximum value, the constraint is not drawn.

Visualization of Arrival Constraints- The arrival procedures constrain the angle and magnitude of the terminal velocity vector relative to the arrival gate. The in-plane constraints at the arrival gate are visualized in figure 13 . The size of the relative terminal velocity vector is made proportional to the vector length. The arrival constraints are satisfied if this vector is within the solid arrival arc. This arc is specified by the arc center angle $\delta_{0}$, the arc aperture $\delta$, and the arc radius $\eta$. The arrival arc is visualized at all times. The scalefactor for visualizing the terminal velocity in this example is $500 \mathrm{~m}$ length per $1 \mathrm{~m} / \mathrm{sec}$.

The out-of-plane limits on the terminal approach velocity are depicted in figure 11. The approach velocity has to be within the constraint brackets. If the velocity is less than $80 \%$ of the allowed maximum value, the constraint is not drawn.

Visualization of Plume Impingement Constraints- Plume impingement constraints limit the magnitude and direction of a maneuvering burn. The in-plane impingement constraints of a burn given at a way point toward the target are illustrated in figure 14. The burn-vector symbol, whose size is proportional to the magnitude of the burn, is not allowed to cross the bracketed impingement constraint arc with aperture $\beta$ and radius $\sigma$. The variables $B$ and $\sigma$ are a function of the distance between way point and target $|\underline{\Delta} \underline{x}|=\left|\underline{x}_{T}-\underline{x}\right|$, whose function depends on the characteristics of plume and target. In this example, $B$ is chosen to be constant and $\sigma$ proportional to $|\Delta \underline{x}|$. If the burn vector does not cross the dotted bracketed arc, the constraint is not drawn. The radius of the dotted arc again is $80 \%$ of $\sigma$ and the aperture angle $10^{\circ}$ larger than $\beta$. The scale factor for visualizing the 
burn velocities was again chosen in this example to be $500 \mathrm{~m}$ length per $1 \mathrm{~m} / \mathrm{sec}$ burn.

Visualization of Approach Velocity Constraints- The method of visualizing the relative approach velocity limit is shown in figure 15. The relative approach velocity of the chaser toward the target is given by the vector $\underline{\underline{v}}=\underline{\mathrm{v}}-\underline{\mathrm{v}}_{\mathrm{T}}$. The line-of-sight vector of the chaser toward the target is $\Delta \underline{x}=\underline{x}_{T}-\underline{x}$. The relative approach velocity vector $\underline{v}_{r}$ is the projection of $\underline{\Delta}$ on $\Delta \underline{x}$ and is given by

$$
\underline{v}_{r}=\frac{\Delta \underline{x}}{|\Delta \underline{x}|^{2}}\left(\Delta \underline{v}^{T} \Delta \underline{x}\right)
$$

where $T$ denotes the transpose, or inner product. The limit on $\left|\underline{v}_{r}\right|$ is a function of the distance between chaser and target $|\underline{\Delta x}|$. In this example, a simple proportional relationship has been chosen. Thus, for a given approach velocity $\left|\underline{v}_{r}\right|$, the allowable range $\rho$ can be computed and visualized by a circle centered about the chaser's position. The approach velocity constraint is violated when the target is located within this circle. The circle is visualized when $\rho$ is greater than $80 \%$ of $|\Delta \underline{x}|$.

Orbital Fuel Use- The orbital fuel use is displayed in viewport 4. The orbital fuel is expressed in total velocity change in meters per second rather than by fuel use in kilograms. The actually spent fuel mass depends on the spacecraft and the thruster characteristics and will be proportional to the total velocity change. A fuel dial is shown which indicates the percentage of fuel remaining from the total amount allowed for the mission. The remaining fuel is indicated by a yellow sector, and fuel use in excess of the allowed amount is indicated by this sector turning red. In addition to the fuel dial, the percentage of fuel left and total fuel use are displayed numerically.

Trajectory Time Markers- Time markers are placed at regular intervals along the chaser and the target trajectories, indicating the time after the start of the maneuver. The time marker is a small bar perpendicular to the trajectory. At the end of this bar a number is displayed which indicates the time in minutes after the beginning of the maneuver. It should be noted that in the viewing system mode, the obliquity and range at which a time marker is located might change. When the numbers are written in the orbital plane, they will change with each viewing system change. A worst-case example occurs when the backside of the orbital plane is viewed and the numbers appear in mirror script. This can be prevented by writing the numbers in the plane of the image rather than in the orbital plane. However, a viewing system change will cause the numbers to move with respect to the marker. Special care is given to the automatic repositioning of the numbers after a viewing system change. The numbers should be placed such that they do not clutter the trajectory, and clearly point to the corresponding time marker. 
Computation of the relative trajectories is a time-consuming process, which, if done at each program update, results in an unacceptably low update rate. This results in jerky motions and poor control over the positioning of a way point. This problem can be prevented by disabling the trajectory computations and starting them only after the operator has stopped moving the active way point.

In order to avoid disturbing delays, at each program update interval the $x$ and $y$ output values of the mouse are compared with the values from the previous step. If no change has taken place, a timer is initiated. The trajectory computations are only started $0.3 \mathrm{sec}$ after initiating the timer. After the trajectory is computed, the computed values are stored and displayed and no further computations will take place until the next change in the active way-point position. The $0.3-$ sec delay is essential for ensuring that the operator has completed the positioning process. Often small corrections are made after the way point has been moved but these corrections do not interfere with the program's update rate, because of inhibition of the trajectory calculation. Sometimes subsequent changes are made after the operator has reviewed the position. These changes are seldom made earlier than 0.5 sec after the last change, and this is after the trajectory has been recomputed.

It should be noted that although the trajectory computations are subject to delay, this is not the case with the computation of variables which relate to the way points themselves, such as maneuvering-burn vectors, relative-velocity vectors and operational constraints. The computation of these variables is less timeconsuming and is done at each program-update interval. Continuous update of these variables is essential in order to give the operator immediate feedback of the effect of a certain design action on maneuvering burns or approach velocities.

A second enhancement for minimizing the computational load of the trajectory computations is a variable time-step size algorithm. The trajectory points are computed at fixed intervals of time rather than at fixed distance intervals along the trajectory. Since the trajectory length might vary, the distance between the trajectory points might vary as well. At each program iteration, the total trajectory length is computed and divided by the total trajectory time to obtain an averaged trajectory velocity. The time step is computed by dividing a preset required averaged distance between trajectory points by the averaged trajectory velocity.

\section{CONCLUDING REMARKS}

The proposed interactive orbital planning system should be seen as a preliminary step in determining the display format of graphical trajectory design, which will be useful in the dense Space Station environment. The examples shown here deal with the most general situation, which involves departures from, and arrival at, nonstationary locations. However, most of the co-orbiting spacecraft are likely to be placed on the $\mathrm{V}$-bar, and thus, at stationary positions. Missions with spacecraft 
at nonstationary positions and substantial out-of-plane motion thus represent a worst-case situation, and as shown here are chosen to demonstrate the capabilities of interactive graphical trajectory design, rather than to represent the average type of maneuver to be executed at the Station.

Likewise, it is difficult to predict whether the constraints used here will be relevant and realistic in the future Space Station environment. They predict in a broad sense the types of restrictions which are expected in the multivehicle environment; e.g., limitations on approach rates, plume impingement, and clearance from structures. It is also likely that the future environment will pose different constraints that might originate from the specific character of a mission, such as a specific scenario in which a telescope or manufacturing platform is approached and serviced.

A third restriction of the display relates to the way the orbital maneuvering system is activated. Only pure-impulse maneuvering burns are considered, in which the duration of the burn is negligible with respect to the duration of the mission, and in which these burns cause major changes in the relative trajectories. Stationkeeping or fly-by missions, however, require a more sustained type of activation, such as periodic small burns with intervals of several seconds over a time span of several minutes. A more distributed way of activating the orbital maneuvering system can be introduced in which the operator has control over the frequency and time span of the activation. Ways can be found to enable this type of control to be activated and visualized.

A last restriction relates to the way the spatial trajectory is visualized. The perspective main view shows the projection of the actual trajectory on the orbital plane, rather than the trajectory itself. The reason for this is twofold. The orbital trajectory (with its typical cycloidal shape), when shown without lines projected on the orbital reference plane, is ambiguous and might seem to bend out of the orbital plane. This illusion results from the viewer's familiarity with common objects such as a coil spring, and has first been reported in reference 7 . Therefore, the trajectory cannot be shown without its projection on the orbital plane. Second, the symbolic enhancements and burn vectors relate to the in-plane motion and match with the trajectory projection on the orbital plane. Thus, both the trajectory and its projection should be visualized. However, in a perspective plan view, i.e., viewed along the $y^{0}$ axis, both the trajectory and its projection on the orbital plane will show up as separate curves, which might be highly confusing. Therefore a compromise has been sought, in which the projection is shown together with pedestals placed at the way points and orthogonal to the orbital plane. The end of the pedestal shows the actual three-dimensional position of the way point.

In spite of these restrictions, the proposed display clearly demonstrates the usefulness of interactive graphical trajectory design. The use of the graphical, symbolical, and computational enhancements indicates the direction in which a solution for a multivehicle environment display should be sought. A still unanswered question relates to the degree of automation that should be introduced into the display. Parts of the mission could be performed through the use of optimization techniques; e.g., to find the fuel-optimal way point which clears a spatial 
constraint in part of the mission, or to find a way point which satisfies the terminal constraints. However, since the solution space of a complex situation is virtually infinite, it is doubtful whether this mission can be performed entirely automatically. It is therefore expected that frequently occurring routine operations, such as searching the local solution space for the optimal location of a way point, might be handed over to an optimization scheme. These solutions can be reviewed by the operator and manually changed if necessary.

An experimental program is planned in which operators will carry out a series of design missions which vary in complexity and constraints. In a tutorial session, the operators will be familiarized with the orbital motions, orbital control methods, and operational constraints. Furthermore, the operators will be familiarized with the system control functions of the viewing system motions and way point editing process. Each operator action, i.e., viewing system or trajectory design, will be time-marked and recorded. Statistics of the viewing system actions will show preferred viewing situations for each condition. Review of the trajectory design actions might reveal the existence of heuristic design rules which might be utilized in automated design schemes.

Ames Research Center

National Aeronautics and Space Administration Moffett Field, CA 94035, April 14, 1988 


\section{APPENDIX}

\section{MATHEMATICAL BACKGROUND}

\section{Relative Motion in Space Station Coordinates}

The relative position and velocity of a co-orbiting spacecraft in Space Station coordinates are $\underline{x}^{\circ} \equiv\left\{x^{0}, y^{0}, z^{\circ}\right\}$ and $\underline{x}^{\circ} \equiv\left\{\dot{x}^{\circ}, \dot{y}^{\circ}, \dot{z}^{\circ}\right\}$, and are obtained from Space Station-based measurement equipment. Since the displacements and velocities in the out-of-orbital-plane direction $y^{\circ}$ are usually much smaller than the ones in the orbital plane in the $x^{\circ}$ and $z^{\circ}$ directions, and since an out-of-plane maneuvering burn does not significantly alter the total orbital energy, the out-of-plane motion can be decoupled from the in-plane motion. Hence, the in-plane motion will be analyzed first.

The in-plane orbital situation is shown in figure 16. At the initial time $t=t_{0}$ the radius of the Space Station orbit $R_{0}$ is given by

$$
R_{0}=R_{E}+h
$$

where $R_{E}=6,378,140 \mathrm{~m}$ is the equatorial Earth radius and $\mathrm{h}=480,000 \mathrm{~m}$ is the altitude of the Space Station orbit above the Earth surface. The absolute orbital velocity of the Space Station is then given by

$$
v_{0}=\left(\frac{G M}{R_{0}}\right)^{1 / 2}
$$

where $\mathrm{GM}=3.986005 \cdot 10^{14} \mathrm{~m}^{3} / \mathrm{sec}^{2}$ is the geocentric gravitational constant. For simplicity the curvature of the $V$-bar is assumed to be negligible, so that

$$
\begin{aligned}
& S \approx x^{0} \\
& r \approx-z^{0} \\
& v=\left[(\dot{s})^{2}+(\dot{r})^{2}\right]^{1 / 2} \\
& \alpha=\tan ^{-1}(\dot{r} / \dot{s}) \quad \dot{s} \geq 0 \\
& \alpha=\tan ^{-1}(\dot{\mathrm{r}} / \dot{\mathrm{s}})+180^{\circ} \quad \dot{\mathrm{s}}<0
\end{aligned}
$$

where $\mathbf{s}$ is the distance measured along the $V$-bar between the Space Station and the spacecraft's R-bar; $r$ is the distance of the spacecraft above the V-bar, measured along the R-bar; $v$ is the magnitude of the relative velocity; and $\alpha$ is the angle of its direction, measured from the $V$-bar in an upward direction (positive rotation 
in the right-hand system). The relative velocity reflects the situation just after the activation of a maneuvering burn.

The squared absolute orbital velocity follows from the geometry of figure 16 and is then given by

$$
v^{2}=v_{0}^{2}\left[\left(\frac{R}{R_{0}}\right)^{2}+2\left(\frac{R}{R_{0}}\right)\left(\frac{v}{v_{0}}\right) \cos \alpha+\left(\frac{v}{v_{0}}\right)^{2}\right]
$$

The orbital energy equation can be written as

$$
\frac{1}{\mathrm{a}}=\frac{2}{\mathrm{R}}-\frac{\mathrm{V}^{2}}{\mathrm{GM}}
$$

where $a$ is the semimajor axis of the spacecraft orbit. Substituting (A5) into (A6) and using (A2) yields

$$
\left(\frac{\mathrm{R}}{\mathrm{a}}\right)=2-\left[\left(\frac{\mathrm{R}}{\mathrm{R}_{\mathrm{o}}}\right)^{3}+2\left(\frac{\mathrm{R}}{\mathrm{R}_{\mathrm{o}}}\right)^{2}\left(\frac{\mathrm{v}}{\mathrm{V}_{\mathrm{o}}}\right) \cos \alpha+\left(\frac{\mathrm{R}}{\mathrm{R}_{\mathrm{o}}}\right)\left(\frac{\mathrm{v}}{\mathrm{V}_{\mathrm{o}}}\right)^{2}\right]
$$

and using the fact that $R=R_{0}+r$ yields

$$
\begin{aligned}
\left(\frac{R}{a}\right)= & 1-2\left(\frac{v}{V_{0}}\right) \cos \alpha-\left(\frac{v}{V_{0}}\right)^{2}-\left(\frac{r}{R_{0}}\right)\left[3+4\left(\frac{v}{V_{0}}\right) \cos \alpha+\left(\frac{v}{V_{0}}\right)^{2}\right] \\
& -\left(\frac{r}{R_{0}}\right)^{2}\left[3+2\left(\frac{v}{V_{o}}\right) \cos \alpha\right]-\left(\frac{r}{R_{0}}\right)^{3}
\end{aligned}
$$

Note that in equation ( $A 7 b$ ) the ratio $r / R_{0}$ rather than $R / R_{0}$ is used. This is done for reasons of numerical accuracy.

The shape of the orbit is given by

$$
R=\frac{a\left(1-e^{2}\right)}{1+e \cos v}
$$

where $v$ is the true anomaly, which is the angular position in orbit after perigee passage and $e$ the eccentricity of the orbit. Defining the auxiliary angle $E$, the eccentric anomaly, and manipulating the orbital energy equation and the orbital angular momentum equation yields (see ref. 1) 


$$
\begin{gathered}
A=e \cos E=1-\left(\frac{R}{a}\right) \\
B=e \sin E=\left(\frac{V}{V_{0}}\right) \sin \alpha\left[\left(\frac{R}{R_{0}}\right)\left(\frac{R}{a}\right)\right]^{1 / 2}
\end{gathered}
$$

Now, for known $v, \alpha, r, V_{o}$, and $R_{0}$, the values for $A$ and $B$ can be computed by first evaluating $R / a$ with equation ( $A 7 b$ ) and substituting this value in equations $(A 9 a, b)$. The eccentricity follows from

$$
e=\left[A^{2}+B^{2}\right]^{1 / 2}
$$

and $E$ from

$$
\begin{array}{ll}
E=\tan ^{-1}(B / A) & A \geq 0 \\
E=\tan ^{-1}(B / A)+180^{\circ} & A<0
\end{array}
$$

The mean anomaly at time $t=t_{0}$ is computed from Kepler's equation

$$
M\left(t_{0}\right)=n\left(t_{0}-T\right)=E-e \sin E=E-B
$$

where $\mathrm{n}$ is the mean motion or angular orbital rate of the spacecraft in radians per second, given by

$$
n=\left(\frac{G M}{a^{3}}\right)^{1 / 2}
$$

and $t=T$ is the time of perigee passage. The mean motion $n_{0}$ of the Space Station is given by

$$
\mathrm{n}_{\mathrm{o}}={\frac{\mathrm{GM}}{\mathrm{R}_{\mathrm{o}}^{3}}}^{1 / 2}
$$

Combining equations (A13) and (A14) yields

$$
n=n_{0} c^{3 / 2}
$$

where

$$
C=\frac{(R / a)}{\left(R / R_{0}\right)}
$$

and the difference in mean motion $\Delta n=n-n_{0}$ between spacecraft and Space Station is approximated by 


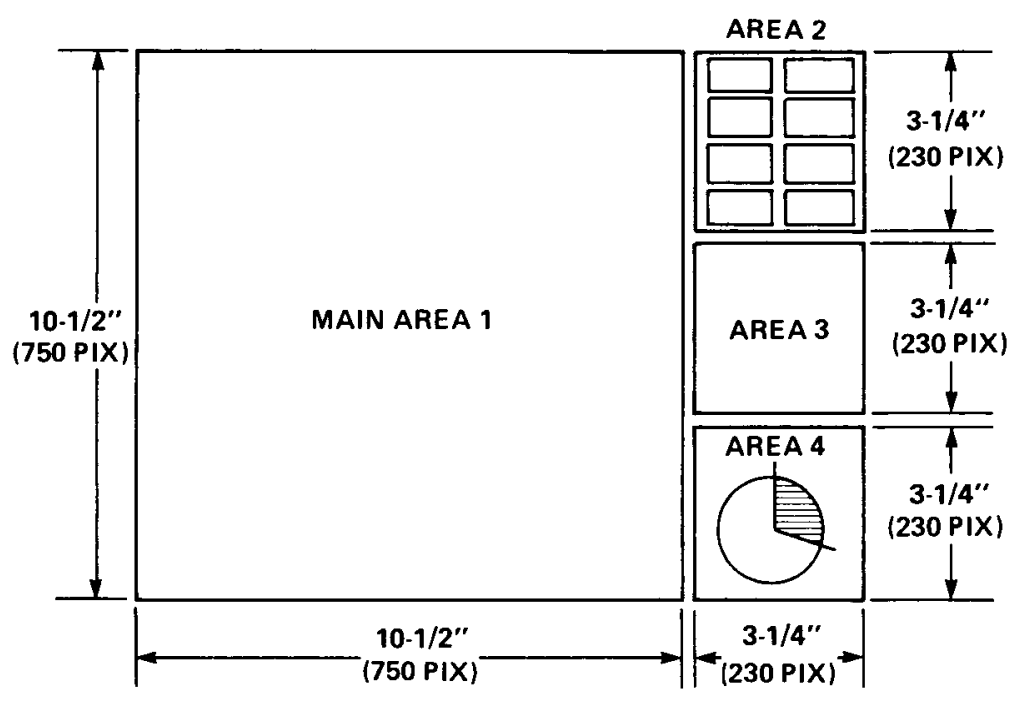

Figure 8.- Layout of the display area.

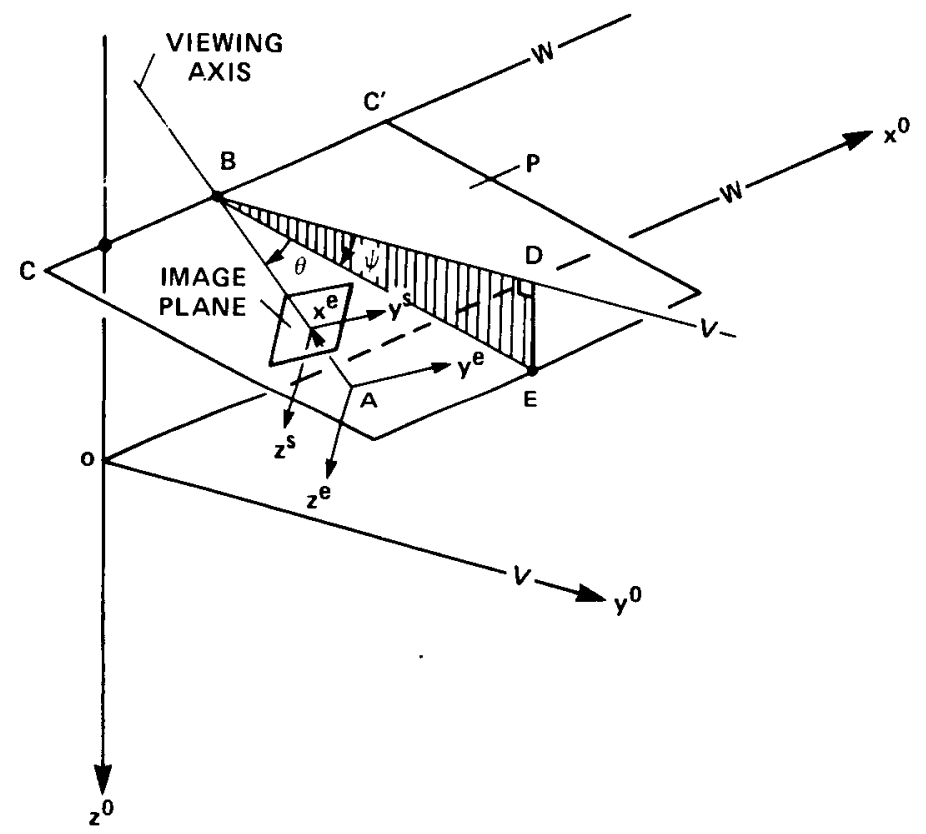

Figure 9.- Geometry of the viewing situation. 
(a)

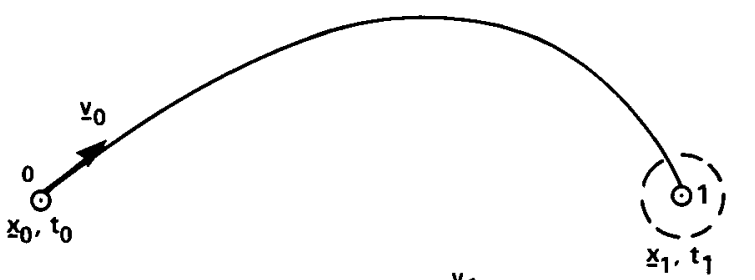

(b)
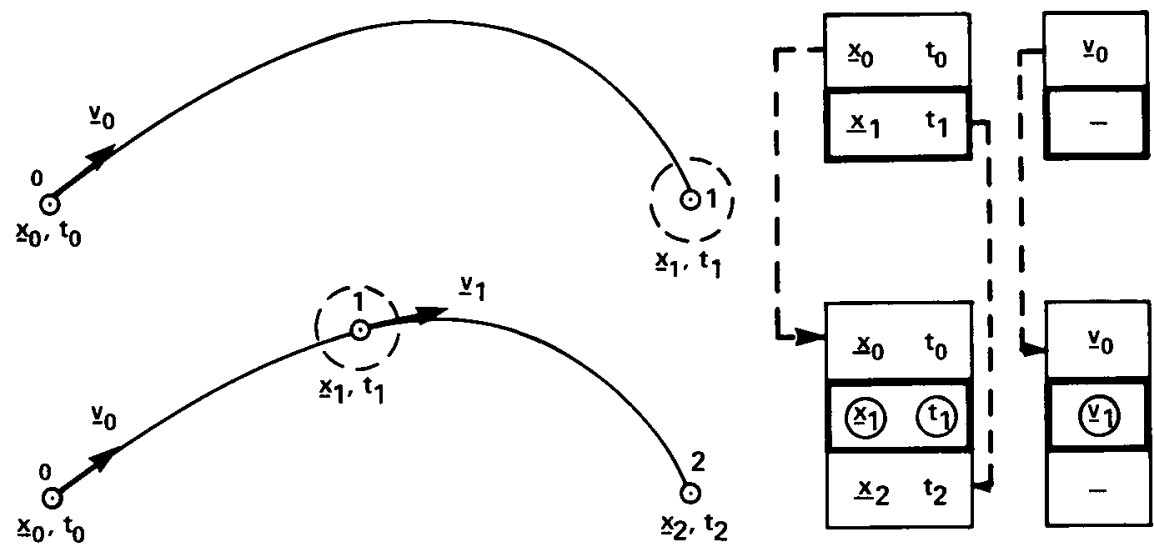

(c)
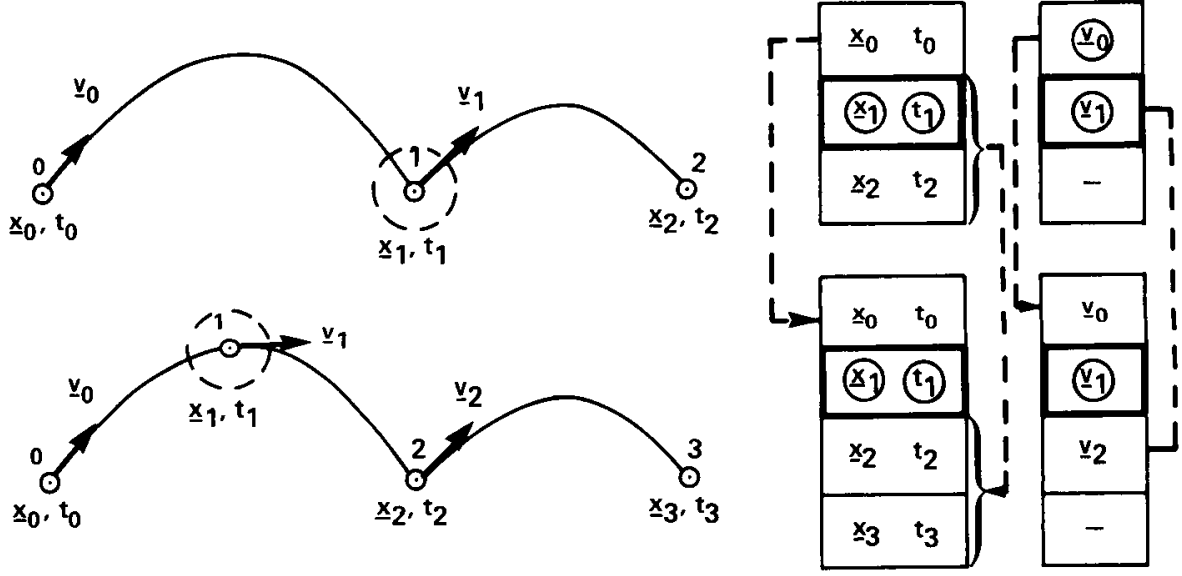

(d)

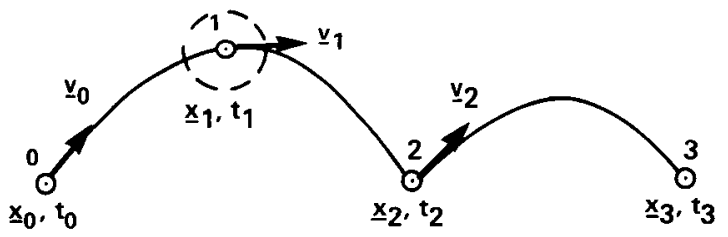

(e)
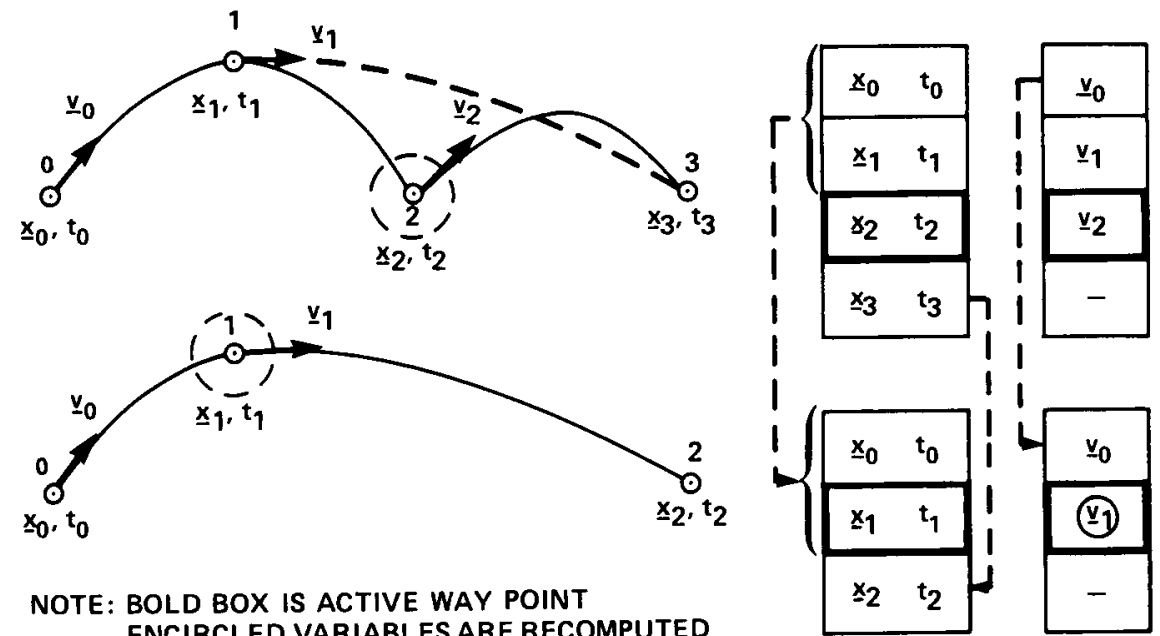

NOTE: BOLD BOX IS ACTIVE WAY POINT ENCIRCLED VARIABLES ARE RECOMPUTED

Figure 7.- Editing of way points. 
POSITIVE V-BAR BURN

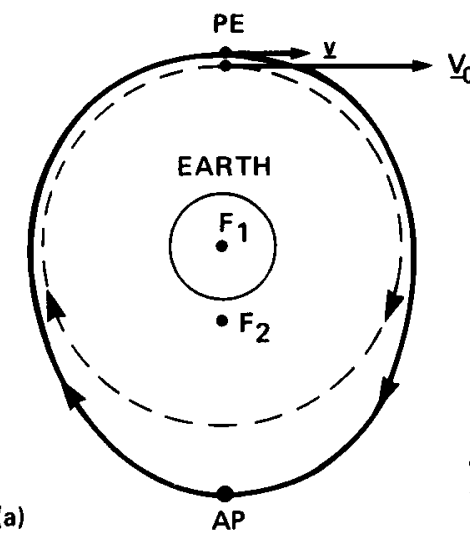

NEGATIVE V-BAR BURN

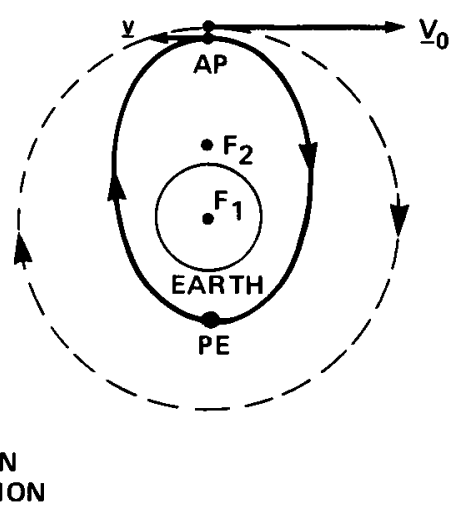

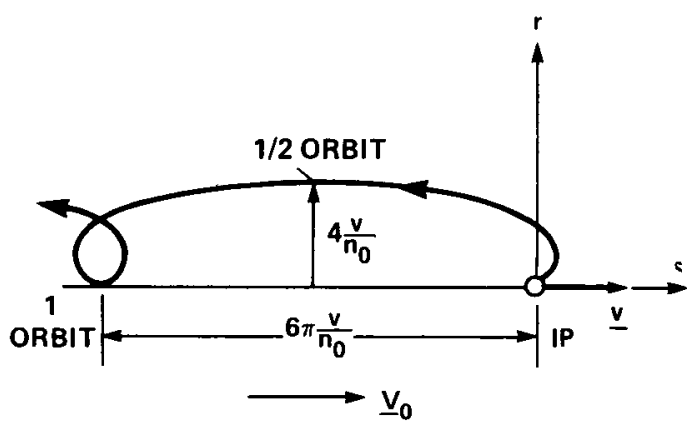

(b)

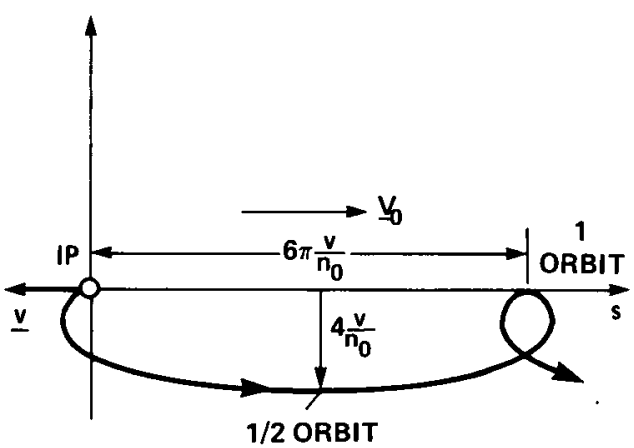

1/2 ORBIT

Figure 5.- Orbital motion after V-bar burn. (a) Shape of the orbit.

(b) Trajectory relative to the Space Station.

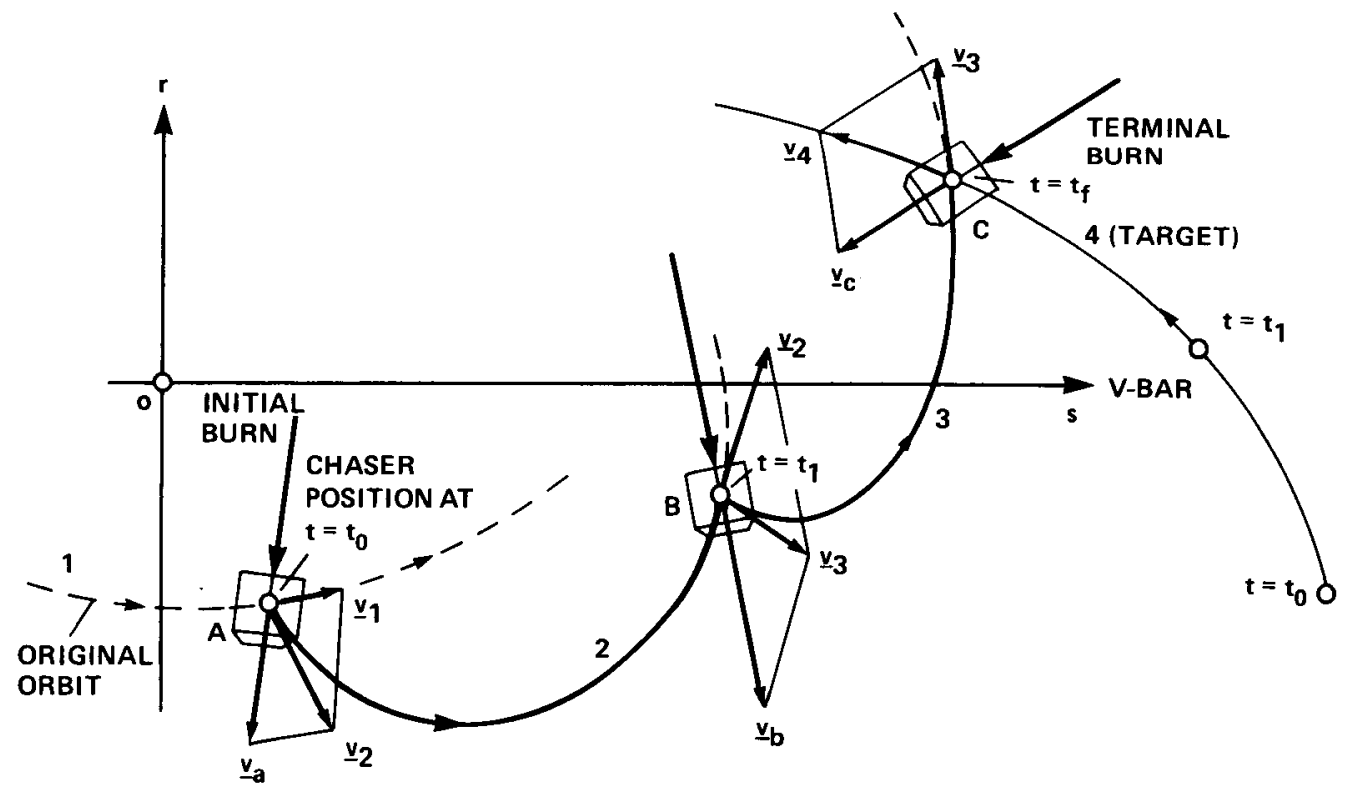

Figure 6.- Example of a three-burn maneuver. 

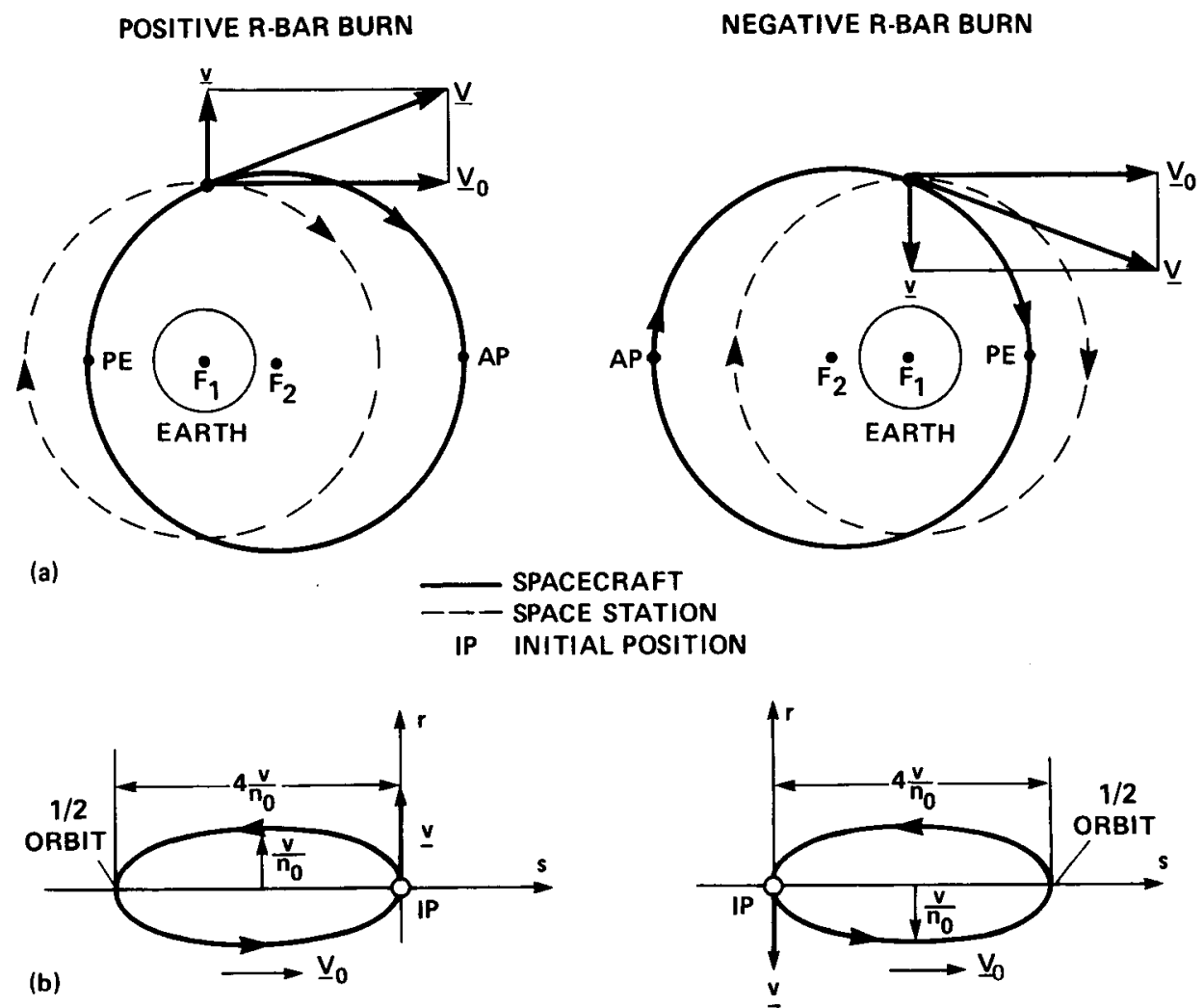

Figure 4.- Orbital motion after R-bar burn. (a) Shape of the orbit. (b) Trajectory relative to the Space Station. 
POSITIVE RADIAL SEPARATION

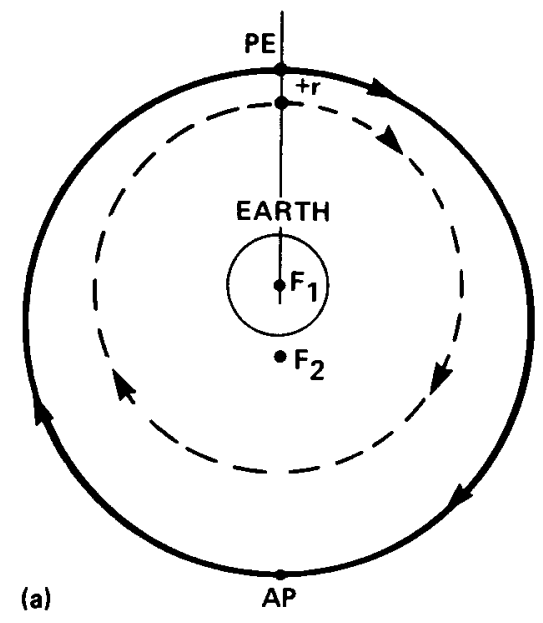

NEGATIVE RADIAL SEPARATION
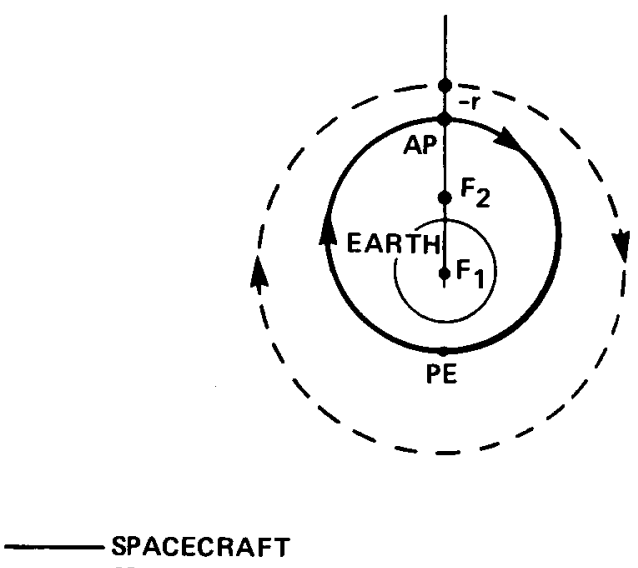

- SPACE STATION

IP INITIAL POSITION

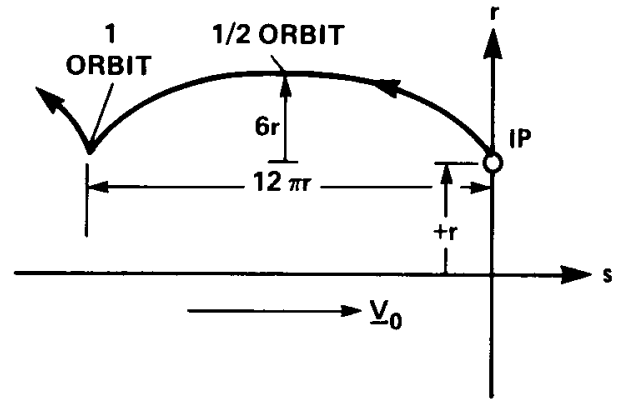

(b)

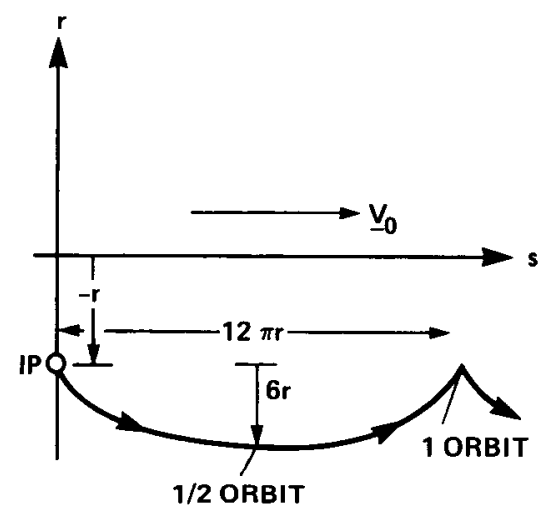

Figure 3.- Orbital motion for an initially stationary spacecraft with radial separation. (a) Shape of the orbit. (b) Trajectory relative to the Space Station. 


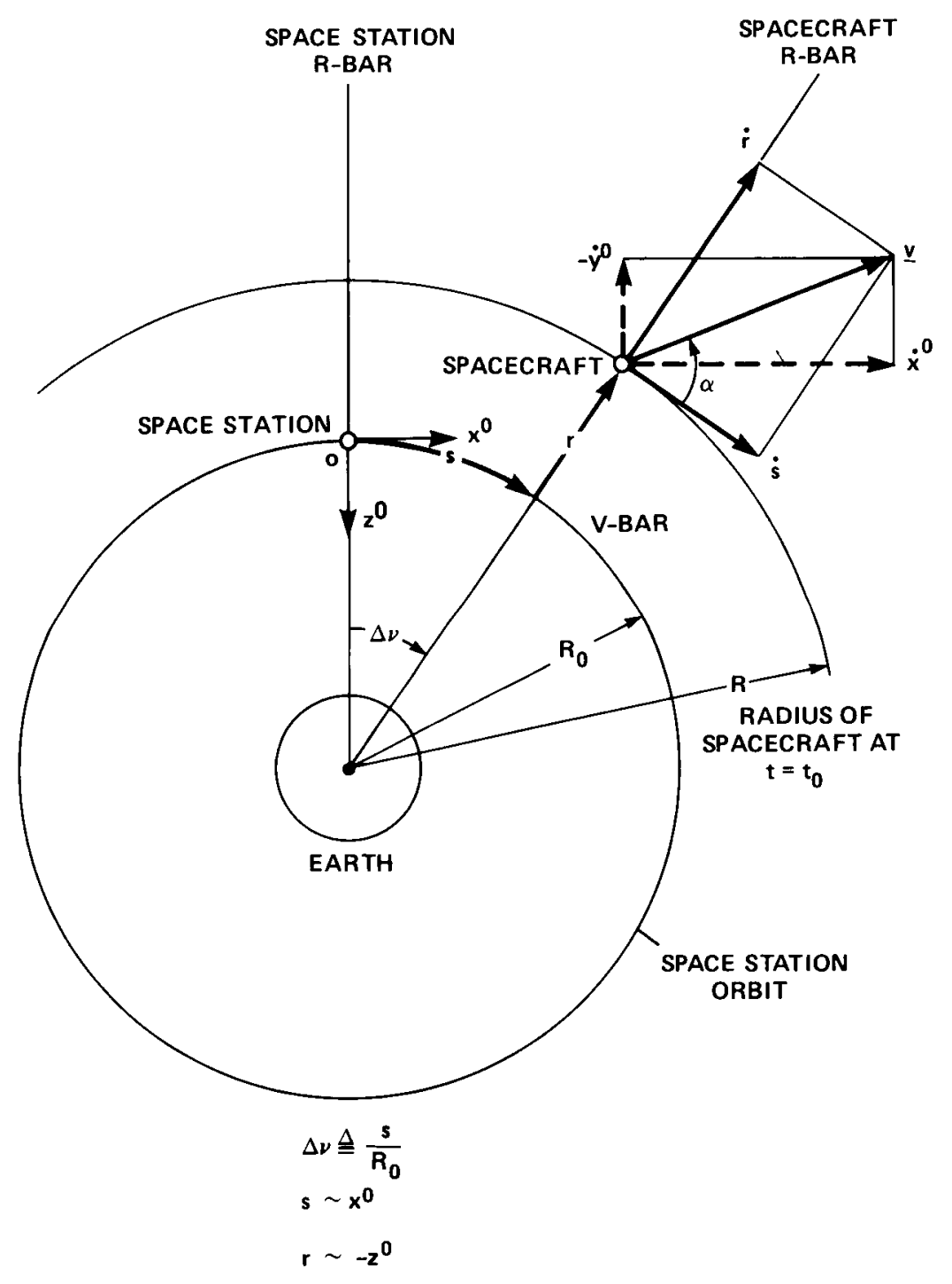

Figure 2.- Definition of coordinate systems. 

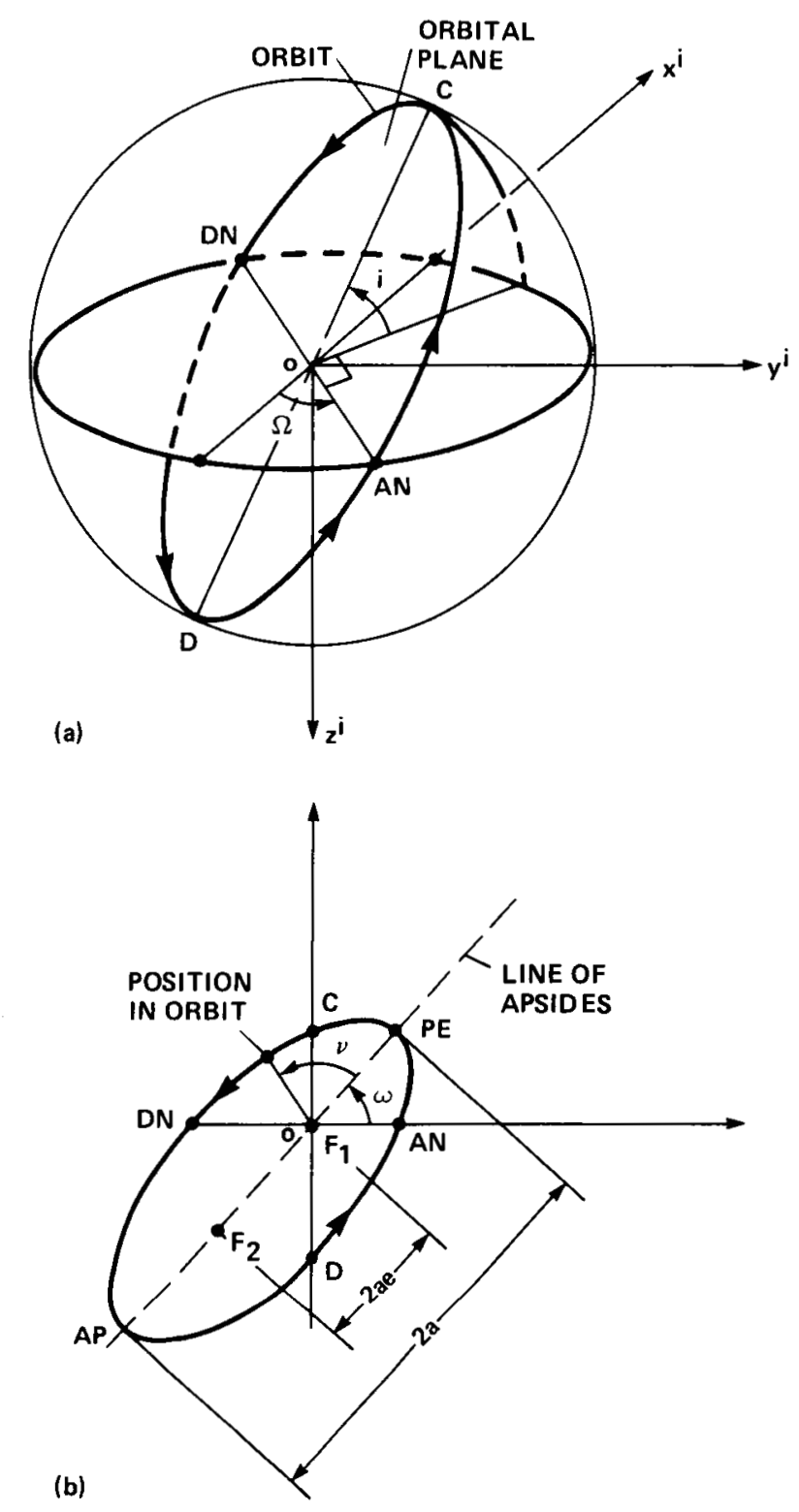

Figure 1.- Definition of the orbit. (a) Orientation of the orbital plane. (b) Shape of the orbit in the orbital plane. 
1. Taff, L. G.: Celestial Mechanics, A Computational Guide for the Practitioner. John Wiley \& Sons, 1985.

2. Kovalevsky, J.: Introduction to Celestial Mechanics. Translated by Express Translation Service, Springer-Verlag New York Inc., New York, 1984.

3. Thomson, W. T.: Introduction to Space Dynamics. Dover Publications, Inc., New York, 1986.

4. NASA Lyndon B. Johnson Space Center: Flight Procedures Handbook. JSC-10566, Nov. 1982.

5. Brody, A. R.: Spacecraft Flight Simulation: A Human Factors Investigation into the Man-Machine Interface between an Astronaut and a Spacecraft Performing Docking Maneuvers and other Proximity Operations. MSc Thesis, Massachusetts Institute of Technology, Apr. 1987.

6. NASA Lyndon B. Johnson Space Center: Rendezvous/Proximity Operations Workbook. RNDZ 2102, Feb. 1985.

7. Ellis, S. R.; and Grunwald, A. J.: A New Illusion of Projected ThreeDimensional Space. NASA TM-100006, July 1987. 


$$
\begin{array}{ll}
F=\left[w\left(t_{0}\right)^{2}+\frac{\dot{w}\left(t_{0}\right)^{2}}{n^{2}}\right]^{1 / 2} & \\
\phi=\tan ^{-1}\left[n w\left(t_{0}\right) / \dot{w}\left(t_{0}\right)\right] & \dot{w}\left(t_{0}\right) \geq 0 \\
\phi=\tan ^{-1}\left[n w\left(t_{0}\right) / \dot{w}\left(t_{0}\right)\right]+1800 & \dot{w}\left(t_{0}\right)<0
\end{array}
$$

Using equation (A47), equations $(A 45 a, b)$ can be reformulated to include the angle $\phi$

$$
\begin{aligned}
& w(t)=F \sin \left[n\left(t-t_{0}\right)+\phi\right] \\
& \dot{w}(t)=F n \cos \left[n\left(t-t_{0}\right)+\phi\right]
\end{aligned}
$$

The relative out-of-plane motion at $t=t$ is then computed by substituting the values of $F$ and $\phi$ in equations $(A 49 a, b)$.

\section{Inverse Computations for Out-of-Plane Motion}

The inverse procedure for out-of-plane motion has a simple closed-form solution. As is the case for in-plane motion, the problem is to find the value of $\dot{w}\left(t_{0}\right)$ which, starting from initial position $w\left(t_{0}\right)$, results in a final position $w(t)$ at $t=t$. Equation (A49a) is written in expanded form

$$
w(t)=F \cos \phi \sin n\left(t-t_{0}\right)+F \sin \phi \cos n\left(t-t_{0}\right)
$$

Substituting equations $(A 46 a, b)$ into equation (A50) and rearranging them yields the required $\dot{w}\left(t_{o}\right)$

$$
\dot{w}\left(t_{0}\right)=n\left[w(t)-w\left(t_{0}\right) \cos n\left(t-t_{0}\right)\right] / \sin n\left(t-t_{0}\right)
$$


$R_{0}$ which are defined by the initial relative position at $t=t_{0}$. The corresponding orbital parameters and relative position are computed with equations (A7-A21).

Next, using equations ( $A 44 a, b$ ) the derivatives of $R / a$ are computed by using the same values of $v$ and $\alpha$ of the previous step as were used in the forward algorithm. The derivatives of $R / a$ are used in equations $(42 a, b)$ and $(43 a, b)$ to compute the derivatives of $A$ and $B$. The derivatives of $a, n, \Delta n, e, E_{0}$, and $M_{0}$ are computed with equations $(A 33-A 41)$. Then, together with the values of $D_{1}$ and $D_{2}$ computed with equations (A28a,b), all computed derivatives are used to compute the derivatives of $D_{1}$ and $D_{2}$ with equations (A32a,b). Finally, all computed values are used in equations $(A 29 a, b)$ to compute the derivatives of $s$ and $v$.

The $2 \times 2$ Jacobian matrix can now be formed. The gradient vector of equation (A24) is computed by simple matrix operations. In the computation of the second-derivative matrix, the second-order term on the right-hand side of equation (A25) can be neglected. The new value of $\underline{u}$ is computed with equation (A23).

\section{Out-of-Plane Motion}

The out-of-plane motion of a co-orbiting spacecraft results from a noncoplanarity between the orbital plane of the spacecraft and the plane of the Space Station. An out-of-plane maneuvering burn alters the relative inclination of the orbital plane of the spacecraft and thus the out-of-plane motion. The out-of-plane displacement $w$ and velocity $\dot{w}$ at time $t$ are described by

$$
\begin{aligned}
& w(t) \equiv y^{0}=F \sin n(t-\tau) \\
& \dot{w}(t) \equiv \dot{y}^{0}=F n \cos n(t-\tau)
\end{aligned}
$$

where $F$ is the maximum out-of-plane deviation from the Space Station orbital plane, $t=\tau$ is the time at which the Space Station orbital plane is crossed in the positive $\mathrm{y}^{0}$ direction, and $\mathrm{n}$ is the mean motion of the orbit. given by

At the initial time $t=t_{0}$ the initial out-of-plane position and velocity are

$$
\begin{aligned}
& w\left(t_{0}\right)=F \sin \phi \\
& \dot{w}\left(t_{0}\right)=F n \cos \phi
\end{aligned}
$$

where $\phi$ is the orbital angle after plane crossing at $t=t_{0}$ defined as

$$
\phi=n\left(t_{0}-\tau\right)
$$

and $\dot{w}\left(t_{0}\right)$ reflects the velocity just after a maneuvering burn at $t=t_{0}$. For a known initial position and velocity, $F$ and $\phi$ can be computed using equations $(A 46 a, b)$ 
Differentiating equation (A40) yields

$$
\frac{\partial a}{\partial x}=-a\left[\frac{\partial(R / a)}{\partial x}\right] /\left(\frac{R}{a}\right)
$$

Derivatives of $A$ and $B$

The derivatives of $A$ and $B$ will be evaluated for $v$ and a separately. Differentiating equation ( $\mathrm{A} 9 \mathrm{a}$ ) yields

$$
\begin{aligned}
\frac{\partial A}{\partial v} & =-\frac{\partial(R / a)}{\partial v} \\
\frac{\partial A}{\partial \alpha} & =-\frac{\partial(R / a)}{\partial \alpha} \\
\frac{\partial B}{\partial v} & =\left(\frac{1}{V_{0}}\right) \sin \alpha\left[\left(\frac{R}{R_{0}}\right)\left(\frac{R}{a}\right)\right]^{1 / 2}+\frac{1}{2} B\left[\frac{\partial(R / a)}{\partial v}\right] /\left(\frac{R}{a}\right) \\
\frac{\partial B}{\partial \alpha} & =\left(\frac{v}{V_{0}}\right) \cos \alpha\left[\left(\frac{R}{R_{0}}\right)\left(\frac{R}{a}\right)\right]^{1 / 2}+\frac{1}{2} B\left[\frac{\partial(R / a)}{\partial \alpha}\right] /\left(\frac{R}{a}\right)
\end{aligned}
$$

Derivatives of $\mathrm{R} / \mathrm{a}$

The derivatives of $R / a$ are obtained by differentiating equation (A7).

$$
\begin{aligned}
& \frac{\partial(R / a)}{\partial v}=-\frac{2}{V_{0}}\left(\frac{R}{R_{0}}\right)^{2} \cos \alpha+\left(\frac{R}{R_{0}}\right)\left(\frac{v}{V_{0}}\right) \\
& \frac{\partial(R / a)}{\partial \alpha}=+2\left(\frac{R}{R_{0}}\right)^{2}\left(\frac{v}{V_{0}}\right) \sin \alpha
\end{aligned}
$$

Implementation of the Inverse Algorithm

The inverse algorithm is easily implemented by computing the derivatives and dependencies in the reversed order as presented here. Computer coding can be highly simplified by identifying compound terms which appear more than once in the process. These terms have to be computed only once and their values are substituted whenever necessary. The above equations are presented in a format which makes identification of these terms easier.

The algorithm starts with the computations of the forward algorithm, i.e., based on the values of $v$ and $\alpha$ of the previous step and on the values of $R$ and 
The $t$ derivative of $E_{0}$ involves the derivative of an arc tangent. The general form for this derivative is given by

$$
\frac{\partial\left(\tan ^{-1} z\right)}{\partial x}=\frac{1}{1+z^{2}} \frac{\partial z}{\partial x}
$$

Substituting $B / A$ for $z$ in equation (A36) and using equation (A10) yields

$$
\frac{\partial E_{0}}{\partial x}=\frac{1}{e^{2}}\left[\frac{\partial B}{\partial x} A-B \frac{\partial A}{\partial x}\right]
$$

Thus, evaluating the derivative of $M_{0}$ also requires the derivatives of $A$ and $B$. These derivatives will be evaluated later.

\section{Derivatives of $e$}

The expression for the derivatives of $e$ is obtained by differentiating equation (A10)

$$
\frac{\partial e}{\partial x}=\frac{1}{e} \quad A\left[\frac{\partial A}{\partial x}+B \frac{\partial B}{\partial x}\right]
$$

\section{Derivatives of $\mathrm{n}$ and $\Delta \mathrm{n}$}

The expression for the derivatives of $n$ and $\Delta \mathrm{n}$ is obtained by differentiating equation ( $A 15 a)$. Note that the term $R / R_{0}$ does not depend on $v$ or $a$, since $R=R_{0}+r$ is the radius of the orbit at $t=t_{0}$. This is in contrast with the term $\mathrm{R} / \mathrm{a}$ in which a (which determines the shape of the orbit) depends on $v$ and a. Since $n_{0}$ is invariant, the derivatives of $n$ and $\Delta n$ are equivalent and are given by

$$
\frac{\partial n}{\partial x} \equiv \frac{\partial \Delta n}{\partial x}=1.5 n\left[\frac{\partial(R / a)}{\partial x}\right] /\left(\frac{R}{a}\right)
$$

The derivatives of the term $\mathrm{R} / \mathrm{a}$ will be evaluated last.

$$
\text { Derivatives of a }
$$

a is written as

$$
a=R\left(\frac{R}{a}\right)^{-1}
$$


and accordingly

$$
\begin{aligned}
& \frac{\partial \sin M_{1}}{\partial x}=\left[\frac{\partial M_{0}}{\partial x}+\frac{\partial n}{\partial z}\left(t-t_{0}\right)\right] \cos M_{1} \\
& \frac{\partial \sin M_{0}}{\partial x}=\frac{\partial M_{0}}{\partial x} \cos M_{0} \\
& \frac{\partial \cos M_{1}}{\partial x}=-\left[\frac{\partial M_{0}}{\partial x}+\frac{\partial n}{\partial x}\left(t-t_{0}\right)\right] \sin M_{1} \\
& \frac{\partial \cos M_{0}}{\partial x}=-\frac{\partial M_{0}}{\partial x} \sin M_{0}
\end{aligned}
$$

Differentiating equations (A28a,b), substituting equations (A31a-d), and using equations (A28a,b) again yields

$$
\begin{aligned}
& \frac{\partial D_{1}}{\partial x}=-\frac{\partial M_{0}}{\partial x} D_{2}+\frac{\partial n}{\partial x}\left(t-t_{0}\right) \cos M_{1} \\
& \frac{\partial D_{2}}{\partial x}=\frac{\partial M_{0}}{\partial x} D_{1}+\frac{\partial n}{\partial x}\left(t-t_{0}\right) \sin M_{1}
\end{aligned}
$$

Now, in order to evaluate the derivatives of equations (A29-A32), the derivatives of $M_{0}, e, n, \Delta n$, and $a$ are required.

Derivatives of $M_{0}$

$M_{0}$ follows from equation (A12) and is given by

$$
M_{0}=E_{0}-B
$$

where $E_{0}$ is the eccentric anomaly at time $t=t_{0}$. Differentiating equation (A33) yields

$$
\frac{\partial M_{0}}{\partial x}=\frac{\partial E_{0}}{\partial x}-\frac{\partial B}{\partial x}
$$

Since

$$
E_{0}=\tan ^{-1}(B / A)
$$




$$
\frac{\partial \underline{x}}{\partial \underline{u}}=\left[\begin{array}{ll}
\frac{\partial s}{\partial v} & \frac{\partial s}{\partial \alpha} \\
\frac{\partial r}{\partial v} & \frac{\partial r}{\partial \alpha}
\end{array}\right]
$$

The sequence of dependencies for computing the derivatives of the Jacobian matrix of equation (A26) are outlined here.

$$
\text { Derivatives of } \mathbf{s} \text { and } \mathbf{r}
$$

Equations $(A 19 a, b)$ of the relative trajectory can be written as

$$
\begin{aligned}
& s(t)=s\left(t_{0}\right)+R_{0}\left[\Delta n\left(t-t_{0}\right)+2 e D_{1}\right] \\
& r(t)=r\left(t_{0}\right)+a e D_{2}
\end{aligned}
$$

where

$$
\begin{aligned}
& D_{1}=\sin M_{1}-\sin M_{0} \\
& D_{2}=-\cos M_{1}+\cos M_{0}
\end{aligned}
$$

It is clear that each one of the terms on the right-hand side of equations $(A 27 a, b)$ depends on both $v$ and $\alpha$. Accordingly, the derivatives of these elements with respect to $v$ and $a$ have to be computed. Differentiating equations $(A 27 a, b)$ yields

$$
\begin{aligned}
& \frac{\partial s}{\partial x}=R_{0}\left[\frac{\partial \Delta n}{\partial x}\left(t-t_{0}\right)+2 \frac{\partial e}{\partial x} D_{1}+2 e \frac{\partial D_{1}}{\partial x}\right] \\
& \frac{\partial r}{\partial x}=\left[\frac{\partial a}{\partial x} e+a \frac{\partial e}{\partial x}\right] D_{2}+a e \frac{\partial D_{2}}{\partial x}
\end{aligned}
$$

where the symbol $x$ is used to denote either $v$ or $\alpha$.

$$
\text { Derivatives of } D_{1} \text { and } D_{2}
$$

Following equations $(A 21 a, b), M_{1}$ can be written as

$$
M_{1}=n(t-T)=n\left(t_{0}-T\right)+n\left(t-t_{0}\right)=M_{0}+n\left(t-t_{0}\right)
$$


where

$$
\begin{aligned}
& M_{1}=n(t-T) \\
& M_{0}=n\left(t_{0}-T\right)
\end{aligned}
$$

and $T$ is obtained from equation ( $A 12$ ).

\section{The Inverse Algorithm}

It has been shown that in order to give the operator direct control over the position of way points, the relative trajectory equations have to be solved backward; i.e., for a given initial relative location at $t=t_{0}$ and a final location at $t=t$, the corresponding values of $v$ and $\alpha$ at $t=t_{0}$ have to be found. $A$ Newton-Raphson scheme has been chosen to solve this problem.

Define the state of the system at $t=t$ by the vector $\underline{x} \equiv\{s, r\}$ and the control applied at $t=t_{0}$ to bring the system to this state by the vector $\underline{u} \equiv\{v, \alpha\}$. Then equations (A1)-(A21) show that $\underline{x}$ is a sophisticated nonlinear function of $\underline{u}$. The problem is to find $\underline{u}$, which brings $\underline{x}$ to a given reference position $\underline{x}_{r}$. A dismatch score is defined as

$$
J(\underline{u})=\frac{1}{2}\left(\underline{x}-\underline{x}_{r}\right)^{T}\left(\underline{x}-\underline{x}_{r}\right)
$$

For the $i$ th iteration $\underline{u}_{i}$ is computed from $\underline{u}_{i-1}$ according to

$$
\underline{u}_{i}=\underline{u}_{i-1}-\left.\left[\frac{\partial^{2} J}{\partial \underline{u}^{2}}\right]_{\underline{u}_{i-1}}^{-1} \frac{\partial J}{\partial \underline{u}}\right|_{\underline{u}_{i-1}}
$$

The gradient vector is given by

$$
\left.\frac{\partial J}{\partial \underline{u}}\right|_{\underline{u}_{i-1}}=\left.\left(\underline{x}-\underline{x}_{r}\right)^{T} \frac{\partial \underline{x}}{\partial \underline{u}}\right|_{\underline{u}_{i-1}}
$$

and the $2 \times 2$ second-derivative matrix is given by

$$
\left.\frac{\partial^{2} \mathrm{~J}}{\partial \underline{u}^{2}}\right|_{\underline{u}_{i-1}}=\left.\left(\frac{\partial \underline{x}}{\partial \underline{u}}\right)^{T} \frac{\partial \underline{x}}{\partial \underline{u}}\right|_{\underline{u}_{i-1}}+\left.\left(\underline{x}-\underline{x}_{r}\right)^{T} \frac{\partial^{2} \underline{x}}{\partial \underline{u}^{2}}\right|_{\underline{u}_{i-1}}
$$

Both the gradient vector and the second-derivative matrix require the computation of the $2 \times 2$ Jacobian matrix, which is given by 


$$
\Delta \mathrm{n} \approx-1.5 \mathrm{n}(1-\mathrm{C})
$$

Note that for numerical reasons it is preferred to use a first-order Taylor series approximation in the computation of $\Delta \mathrm{n}$.

It is clear from the above derivations that for a given initial relative position and velocity at $t=t_{0}$, the orbital parameter set, which characterizes the shape of the orbit, can be fully determined. This parameter set can now be used to compute the relative position and velocity at $t$ ime $t=t$. This is done in the following steps. First, the mean anomaly at time $t$ is computed from

$$
M(t)=M\left(t_{0}\right)+n\left(t-t_{0}\right)
$$

Now, Kepler's equation (eq. (12)) can again be employed to compute the eccentric anomaly at time $t=t, E(t)$. However, a simple closed-form solution for solving $E$ does not exist. The common practice in classical celestial mechanics is to use Fourier-Bessel functions and series expansions (see ref. 1). An alternative is to solve the equation numerically by an iterative process such as Newton's false-root method. Once the eccentric anomally $E(t)$ is computed, the ratio $R / a$ at $t=t$ can be found with equation ( $A 9 a)$. Multiplying the ratio with the known value for $a$ and subtracting $R_{0}$ yields the relative distance $r$. By using equation ( $A 8$ ) the true anomaly $v(t)$ at $t=t$ can be found. The relative distance $s$ is obtained from

$$
s(t)=R_{0}\left[v(t)-v_{0}(t)\right]
$$

where $\nu_{0}$ is the true anomaly of the Space Station.

In this research, the Fourier-Bessel series expansions are used (see ref. 1). Since the eccentricity of the orbit is very small, second-and higher-order terms were dropped. The resulting equations for the relative trajectories and trajectory rates, starting from $t=t_{0}$ are given by

$$
\begin{aligned}
& s(t)=s\left(t_{0}\right)+R_{0}\left[\Delta n\left(t-t_{0}\right)+2 e\left(\sin M_{1}-\sin M_{0}\right)\right] \\
& r(t)=r\left(t_{0}\right)+a e\left(\cos M_{0}-\cos M_{1}\right)
\end{aligned}
$$

and

$$
\begin{aligned}
& \dot{s}(t)=R_{0}\left[\Delta n+2 e n \cos M_{1}\right] \\
& \dot{r}(t)-\text { aen } \sin M_{1}
\end{aligned}
$$




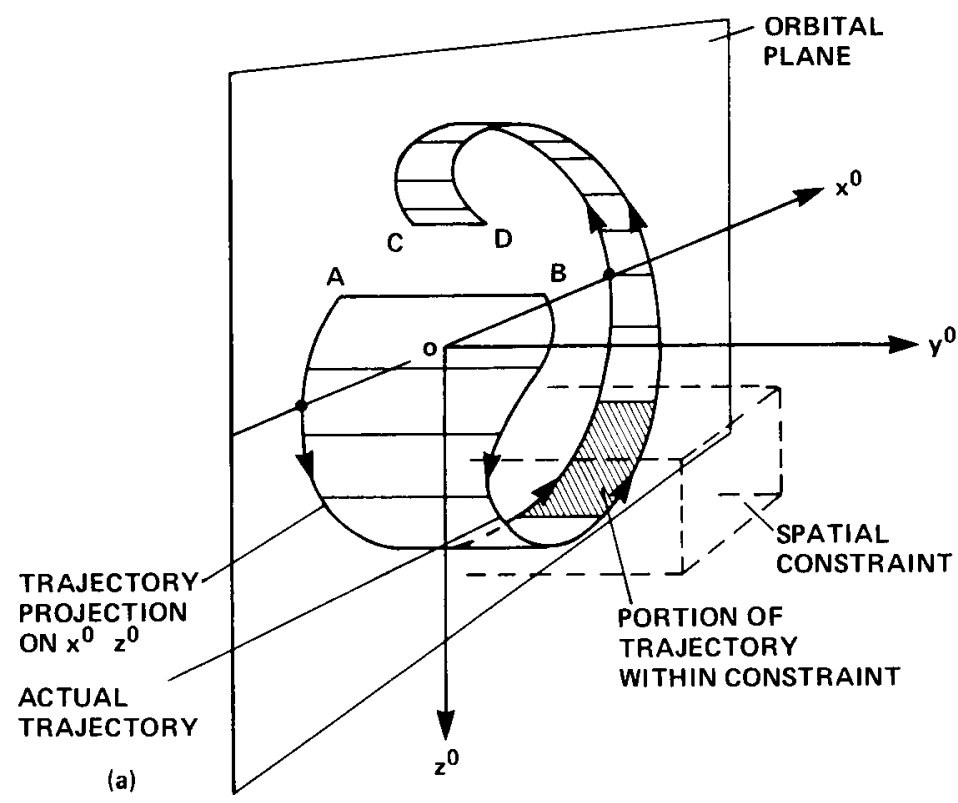

(a)

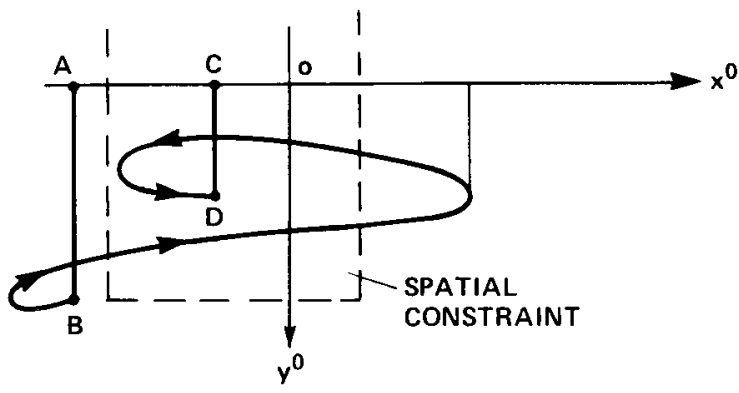

(c)

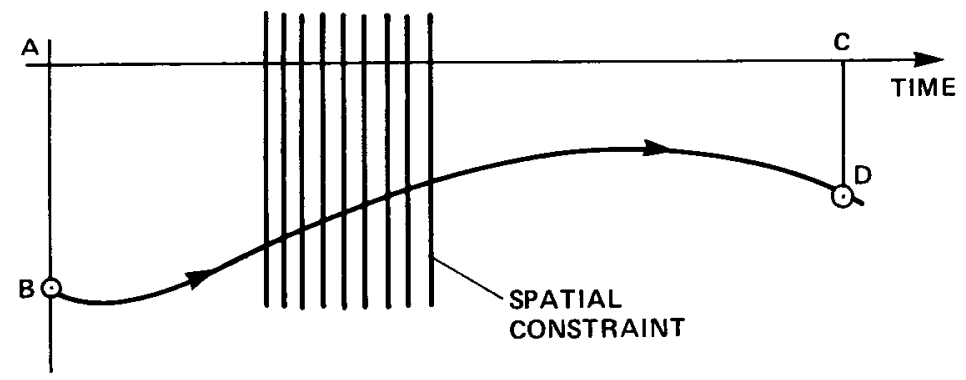

Figure 10.- Principle of the time-axis format. (a) Perspective view.

(b) View along the $z^{0}$ axis. (c) Time-axis format. 


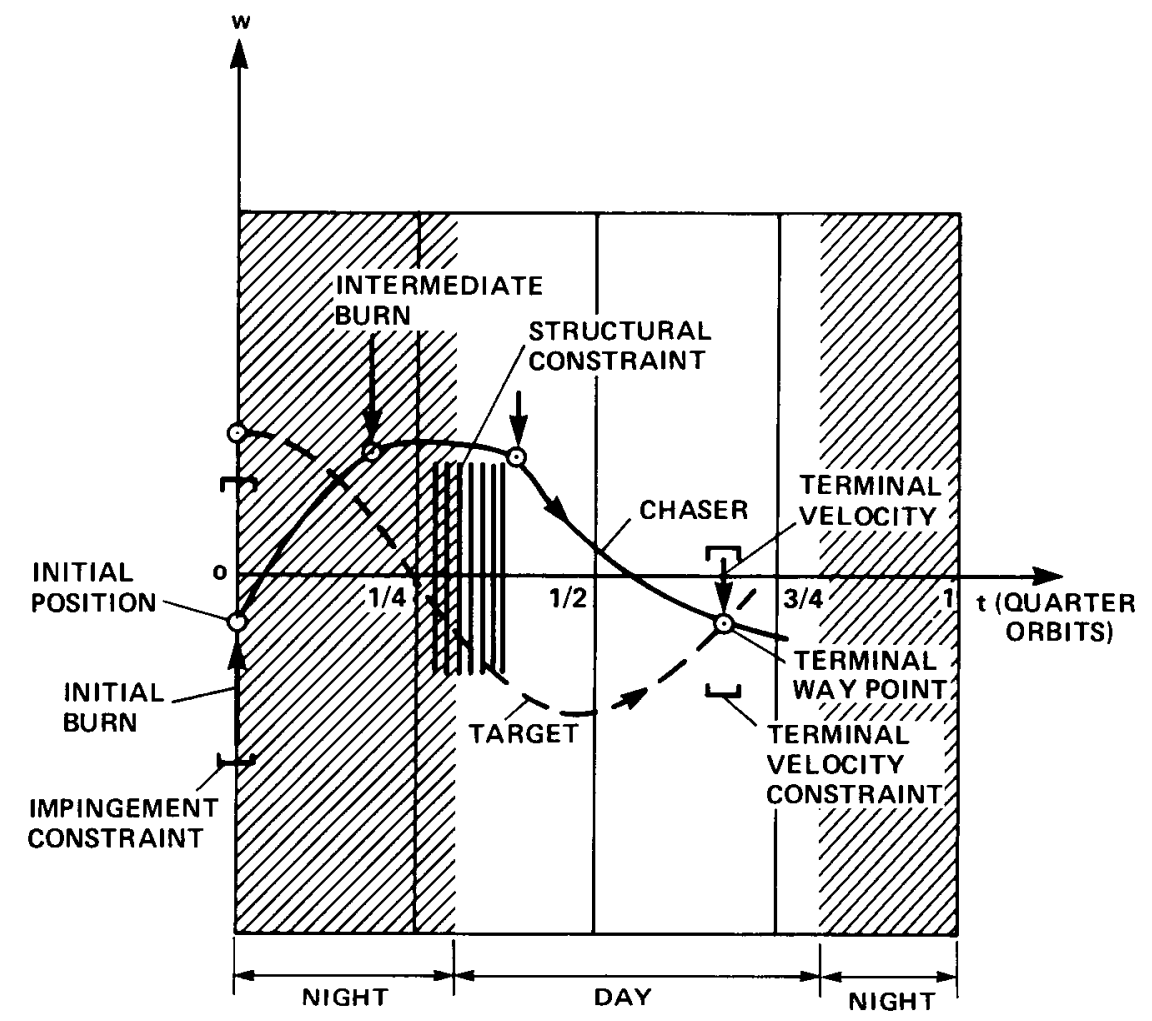

Figure 11.- Time-axis format with operational constraints and day-night indication.

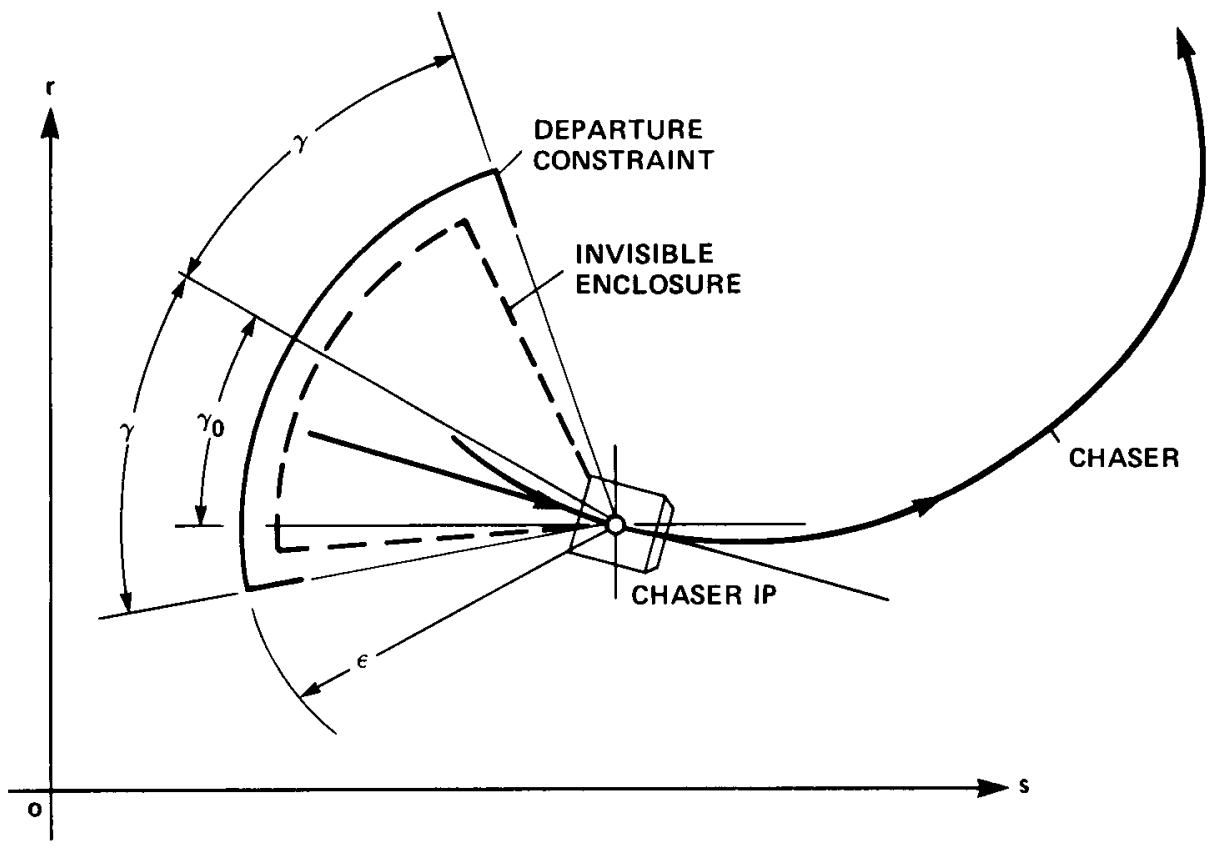

Figure 12.- Departure constraints. 


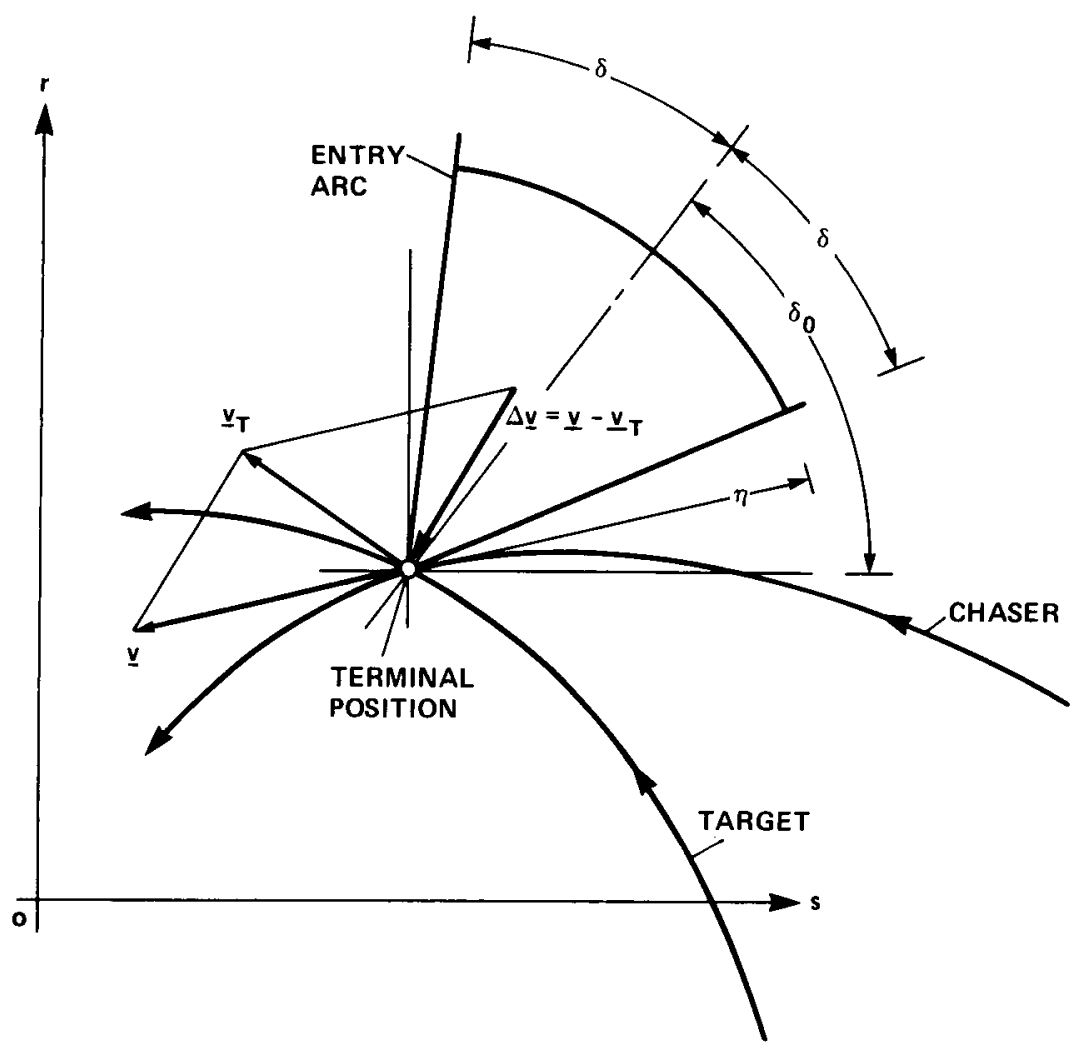

Figure 13.- Arrival constraints.

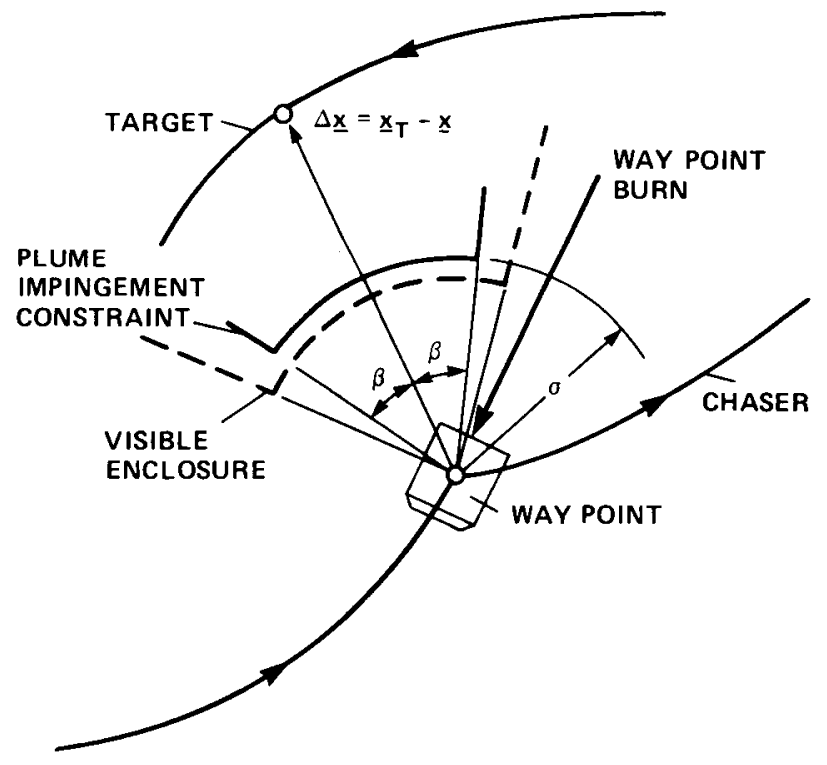

Figure 14.- Plume impingement constraints. 


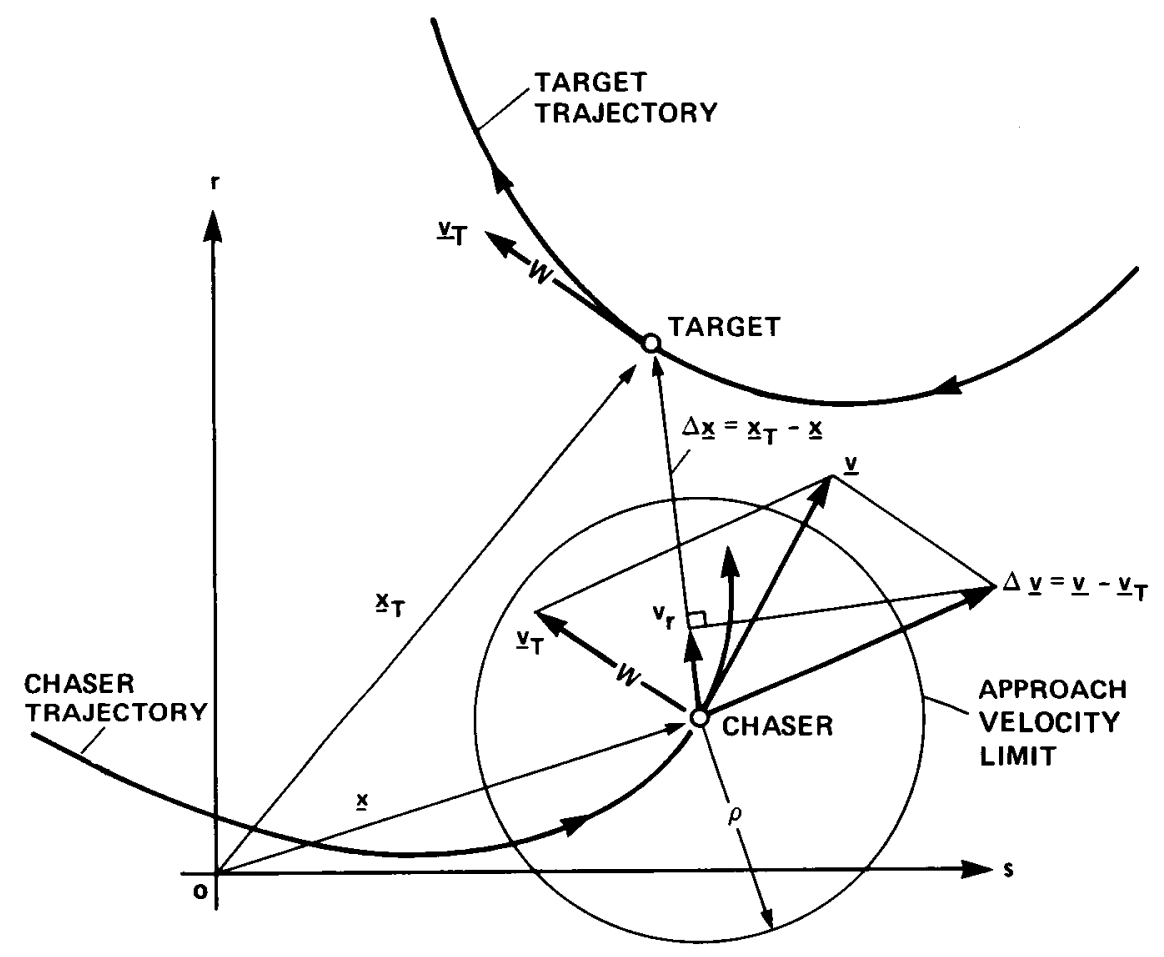

Figure 15.- Approach velocity limit circle. 


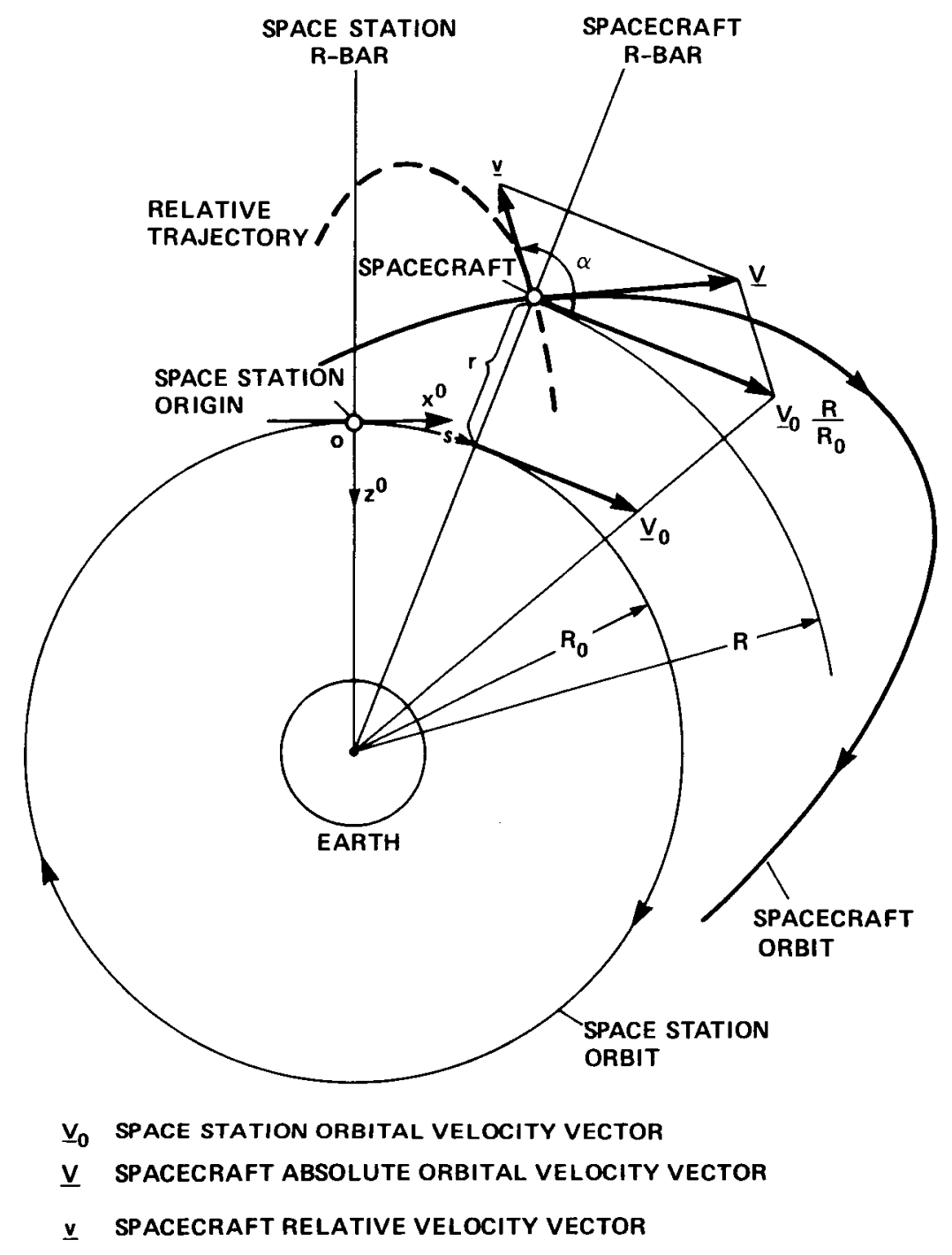

Figure 16.- In-plane orbital situation. 




